\title{
Modeling the Euglycemic Hyperinsulinemic Clamp by Stochastic Differential Equations
}

\author{
Umberto Picchini $^{1} \quad$ Andrea De Gaetano ${ }^{1} \quad$ Susanne Ditlevsen ${ }^{2}$
}

\author{
${ }^{1}$ CNR-IASI BioMatLab, Largo A. Gemelli 8 - 00168, Rome, Italy \\ e-mail: umberto.picchini@biomatematica.it (Umberto Picchini), andrea.degaetano@biomatematica.it \\ (Andrea De Gaetano) \\ 2 Department of Biostatistics, University of Copenhagen, Denmark \\ e-mail: sudi@pubhealth.ku.dk
}

Publication details: Journal of Mathematical Biology, 53(5), 771-796, November 2006 [DOI: $10.1007 / \mathrm{s} 00285-006-0032-z]$. The original publication is available at www.springerlink.com.

\begin{abstract}
The Euglycemic Hyperinsulinemic Clamp (EHC) is the most widely used experimental procedure for the determination of insulin sensitivity. In the present study, sixteen subjects with BMI between 18.5 and $63.6 \mathrm{~kg} / \mathrm{m}^{2}$ have been studied with a long-duration (five hours) EHC. In order to explain the oscillations of glycemia occurring in response to the hyperinsulinization and to the continuous glucose infusion at varying speeds, we first hypothesized a system of ordinary differential equations (ODEs), with limited success. We then extended the model and represented the experiment using a system of stochastic differential equations (SDEs). The latter allow for distinction between (i) random variation imputable to observation error and (ii) system noise (intrinsic variability of the metabolic system), due to a variety of influences which change over time. The stochastic model of the EHC was fitted to data and the system noise was estimated by means of a (simulated) maximum likelihood procedure, for a series of different hypothetical measurement error values. We showed that, for the whole range of reasonable measurement error values: (i) the system noise estimates are non-negligible; and (ii) these estimates are robust to changes in the likely value of the measurement error. Explicit expression of system noise is physiologically relevant in this case, since glucose uptake rate is known to be affected by a host of additive influences, usually neglected when modelling metabolism. While in some of the studied subjects system noise appeared to only marginally affect the dynamics, in others the system appeared to be driven more by the erratic oscillations in tissue glucose transport rather than by the overall glucose-insulin control system. It
\end{abstract}


is possible that the quantitative relevance of the unexpressed effects (system noise) should be considered in other physiological situations, represented so far only with deterministic models.

Keywords: mathematical models, dynamical systems, glucose, insulin, parameter estimation, Monte Carlo methods, simulated maximum likelihood.

\section{Introduction}

With the growing epidemiological importance of insulin resistance states (like obesity and Type 2 Diabetes Mellitus, T2DM) and with the increasing clinical recognition of the impact of the so-called metabolic syndrome, the assessment of insulin sensitivity has become a very relevant issue in metabolic research. The experimental procedures currently employed to gather information on the degree of insulin resistance of a subject are the Oral Glucose Tolerance Test (OGTT), the Intra-Venous Glucose Tolerance Test (IVGTT), the Euglycemic Hyperinsulinemic Clamp (EHC), the Hyperglycemic Clamp, the insulin-induced hypoglycemia test $\left(K_{I T T}\right)$, and less commonly used methods based on tracer administration (Ferrannini and Mari (1998), Starke (1992), Wallace and Matthews (2002)). Of these, the EHC is considered the tool of choice in the diabetological community, in spite of its labor-intensive execution, due to the simple interpretation which is usually attributed to the obtained results (DeFronzo et al. (1979), Zierler (1999)). The favor with which the EHC is viewed in this context stems in part from the belief that while mathematical models of the glucose insulin system make untenable assumptions, the EHC approach is relatively assumption-free, or model independent.

In the present work we study the dynamic behavior of glycemia and insulinemia recorded from human subjects during a EHC procedure. We firstly hypothesize a system of ODEs explaining this dynamics (inspired by a previously published deterministic model of the EHC Picchini et al. (2005)), and obtain the corresponding parameter estimates by numerically fitting the model to observed data. This simply deterministic model, however, does not accommodate random variations of metabolism. In fact a deterministic model assumes that: (i) the mathematical process generating the observed glycemias is smooth (continuous and continuously differentiable) in the considered time-frame; and (ii) the variability of the actual measurements is due to observation error, which does not influence the course of the underlying process. An alternative, stochastic, approach would result from the hypothesis that the underlying mathematical process itself is not smooth, at least when considered at the practicable time resolution. The glucose metabolizing organs and tissues are in fact subject to a variety of internal and external influences, which change over time (e.g. blood flow, energy requirements, hormone levels, the cellular metabolism of the tissues themselves) and which may affect the instantaneous glycemias. This second approach would maintain that some degree of randomness is already present in the glucose disposition process itself, and that observational error is superimposed to it. A natural extension of the deterministic model is given by a stochastic differential equations (SDEs) model (see e.g. Kloeden and Platen (1992) and $\varnothing$ ksendal (2000)). We therefore define an SDE model by adding a suitable system variability to the simple deterministic model. 
While SDE models are currently being employed in different applied fields (e.g. finance, engineering, physics), they are rarely used in biomedicine (except for specific fields like neuronal modelling, population growth models and recent contributions, e.g. Tornøe et al. (2004) for another approach to SDE modeling of the EHC), even though it is generally recognized that biological data are fraught with many sources of intrinsic (system) error. This large amount of variability is often attributed to observation error exclusively, giving rise to inaccurate, but manageable, modelling representations of physiological phenomena.

The present work has two main goals: on one hand it purports to determine whether, in a particular physiological situation, system error is identifiable and necessary to explain observations, above and beyond commonly accepted levels of measurement error. The second goal is to show, by means of a practically occurring experimental situation, that SDE models are physiologically relevant and that their parameters can be numerically estimated using commonly available resources.

\section{Material and methods}

\section{$2.1 \quad$ Subjects}

Sixteen subjects were enrolled in the study, 8 normal volunteers and 8 patients from the Obesity Outpatient Clinic of the Department of Internal Medicine at the Catholic University School of Medicine. For one normal subject the recorded glycemia values were accidentally lost and this subject was therefore discarded from the following considerations. The subjects had widely differing BMIs (from 18.5 to $63.6 \mathrm{~kg} / \mathrm{m}^{2}$ ). All subjects were clinically euthyroid, had no evidence of diabetes mellitus, hyperlipidemia, or renal, cardiac or hepatic dysfunction and were undergoing no drug treatments that could have affected carbohydrate or insulin metabolism. The subjects consumed a weight-maintaining diet consisting of at least $250 \mathrm{~g}$. of carbohydrate per day for 1 week before the study. Table 1 reports anthropometric characteristics $(B M I$, $B S A)$ of the subjects, measured plasma glucose and insulin concentrations $\left(G_{\text {fast }}, I_{\text {fast }}\right)$ immediately before the EHC procedure, and the average levels of insulin after $80 \mathrm{~min}$. of clamp insulinization $\left(I_{\max }\right)$. The study protocol followed the guidelines of the Medical Ethics Committee of the Catholic University of Rome Medical School; written informed consent was obtained from all subjects.

\section{$2.2 \quad$ Experimental protocol}

Each subject was studied in the postabsorptive state after a 12-14 h. overnight fast. Subjects were admitted to the Department of Metabolic Diseases at the Catholic University School of Medicine in Rome the evening before the study. At 07.00 hours on the following morning, the infusion catheter was inserted into an antecubital vein; the sampling catheter was introduced in the contralateral dorsal hand vein and this hand was kept in a heated box $\left(60^{\circ} \mathrm{C}\right)$ in order to obtain arterialized blood. A basal blood sample was obtained in which insulin and glucose levels were measured. At 08.00 hours, after a 12-14 h. overnight fast, the EHC was performed according to DeFronzo et al. (1979). A priming dose of short-acting human insulin was given during the initial $10 \mathrm{~min}$. in a logarithmically decreasing manner so that the plasma insulin was raised acutely to the desired level. During the five-hour clamp procedure, the glucose and insulin levels were monitored every $5 \mathrm{~min}$. and every $20 \mathrm{~min}$. respectively, and the rate of infusion of a $20 \%$ glucose solution was adjusted during the procedure following the published 
algorithm DeFronzo et al. (1979). Because serum potassium levels tend to fall during this procedure, $\mathrm{KCl}$ was given at a rate of $15-20 \mathrm{mEq} / \mathrm{h}$ to maintain the serum potassium between 3.5 and $4.5 \mathrm{mEq} / \mathrm{l}$. Serum glucose was measured by the glucose oxidase method using a Beckman Glucose Analyzer II (Beckman Instruments, Fullerton, Calif., USA). Plasma insulin was measured by microparticle enzyme immunoassay (Abbott Imx, Pasadena, Calif., USA).

\subsection{Deterministic model}

In order to explain the oscillations of glycemia occurring in response to hyperinsulinization and to continuous glucose infusion at varying speeds, we hypothesized the following system (inspired by a previously published deterministic model of the EHC Picchini et al. (2005)):

$$
\begin{aligned}
\frac{d G(t)}{d t} & =\frac{\left(T_{g x}\left(t-\tau_{g}\right)+T_{g h}(t)\right)}{V_{g}}-T_{x g} \frac{G(t)}{0.1+G(t)}-K_{x g I} G(t) I(t) \\
\frac{d I(t)}{d t} & =\frac{\left(T_{i G} G(t)+T_{i x}(t)\right)}{V_{i}}-K_{x i} I(t) \\
T_{g h}(t) & =T_{\text {ghmax }} \exp (-\lambda G(t) I(t))
\end{aligned}
$$

where

$$
\begin{gathered}
G(0)=G_{b}, \quad I(0)=I_{b}, \\
T_{g h}(0)=T_{g h b}=T_{g h \max } \exp \left(-\lambda G_{b} I_{b}\right), \\
T_{g x}(s)=0 \quad \forall s \in\left[-\tau_{g}, 0\right] \quad \text { and } \quad T_{i x}(0)=T_{i x b}
\end{gathered}
$$

$T_{g x}(t)$ and $T_{i x}(t)$ are (input or forcing) state variables of which the values are known at each time; the state variables and the parameters are defined in Table 2 and Table 3 . The model is diagrammatically represented in Figure 1.

Equations (1) and (2) express the variations of plasma glucose and plasma insulin concentrations. The variation of glucose concentration in its distribution space is attributed to the external glucose infusion rate, to liver glucose output and to insulin-dependent as well as insulin-independent glucose tissue uptake. Infused glucose raises glycemia after a delay $\tau_{g}$ due to the time required to equilibrate the intravenously infused quantity throughout the distribution space. The insulin-independent glucose tissue uptake process is modelled as a Hill function rapidly increasing to its (asymptotic) maximum value $T_{x g}$; thus for glycemia values appreciably larger than $0.1 \mathrm{mM}$ the insulin-independent glucose tissue uptake is already close to its maximum. This formulation is intended to represent the aggregated apparent zero-order (fixed) glucose utilization mechanism at rest (mainly by the brain and heart Olson and Pessin (1996) and Sacks (1969) p. 320), with the mathematical and physiological requirement that glucose uptake tends to zero as glucose concentration in plasma approaches zero. The variation of insulin concentration in its distribution space (equation (2)) may be thought of as due to the external insulin infusion, to glucose-dependent pancreatic insulin secretion and to the apparently first-order insulin removal from plasma. Equation (3) represents the rate of net Hepatic Glucose Output, starting at maximal HGO at zero glucose and zero insulin and decaying monotonically with increases in both glucose and effective insulin concentrations in the plasma. The net HGO is assumed to be equal to $T_{g h b}$ at the beginning of the experiment and to decrease toward zero as glycemia or insulinemia levels increase. Serum insulin affects glucose clearance through equation (1) and the glucose synthesis rate through equation (3). 
Steady-state conditions are used to decrease the number of free parameters to be estimated: at steady state, before the start of the clamp $\left(G=G_{b}, I=I_{b}, T_{g x}=T_{i x}=0\right)$, we have

$$
\begin{gathered}
T_{g h b}=T_{g h \max } \exp \left(-\lambda G_{b} I_{b}\right) \\
0=\frac{0+T_{g h b}}{V_{g}}-\frac{T_{x g} G_{b}}{0.1+G_{b}}-K_{x g I} I_{b} G_{b} \Rightarrow T_{x g}=\left(\frac{T_{g h b}}{V_{g}}-K_{x g I} I_{b} G_{b}\right) \frac{\left(0.1+G_{b}\right)}{G_{b}} \\
0=\frac{T_{i G} G_{b}+0}{V_{i}}-K_{x i} I_{b} \Rightarrow T_{i G}=\frac{K_{x i} I_{b} V_{i}}{G_{b}}
\end{gathered}
$$

Therefore the parameters $T_{g h b}, T_{x g}$, and $T_{i G}$ are completely determined by the values of the other parameters.

\subsubsection{Deterministic model estimation}

The system (1)-(3) has been numerically integrated by means of a fourth-order Runge-Kutta scheme with constant stepsize equal to $0.5 \mathrm{~min}$. In order to distinguish among the $n$ observations (and corresponding predictions) between glucose and insulin, the indices $j$ for glucose and $k$ for insulin are used as follows: $j \in J, k \in K, J \cap K=\{\varnothing\}, J \cup K=\{1, \ldots, n\}$. We indicate with $\hat{G}(t, \theta) \equiv \hat{G}(t)$ and $\hat{I}(t, \theta) \equiv \hat{I}(t)$ the (numerically integrated) solutions of equations (1)-(3) for parameter $\theta$ at time $t$. The solutions have been fitted by Iteratively ReWeighted Least Squares (IRWLS, see e.g. (Davidian and Giltinan, 1995, chapter 2)) separately on each subject's glycemia and insulinemia time-points, estimating only the free parameters $\theta=\left(G_{b}, I_{b}, K_{x g I}, K_{x i}, T_{g h \max }, V_{g}, V_{i}, \tau_{g}, \lambda\right)$ by minimizing the following loss function

$$
(y-\hat{y})^{\prime} \Omega(y-\hat{y})
$$

where $y$ is the $n \times 1$ array containing both glycemias and insulinemias, observed at times $0=t_{1} \leq t_{2} \leq \cdots \leq t_{n} ; \hat{y}$ is the array of corresponding predictions obtained by numerical integration of the system (1)-(3), $\hat{y}\left(t_{j}\right)=\hat{y}_{j}=\hat{G}\left(t_{j}\right) \forall j \in J, \hat{y}\left(t_{k}\right)=\hat{y}_{k}=\hat{I}\left(t_{k}\right) \forall k \in K ; \Omega$ is an $n \times n$ diagonal matrix of weights. Here $Z^{\prime}$ denotes the transpose of the matrix $Z$. The statistical weight associated with a generic glucose concentration point $y_{j}$ has been defined as $1 /\left(\hat{y}_{j} C V_{G}\right)^{2}$, where $C V_{G}$ is the coefficient of variation for glucose. Similarly the statistical weight associated with a generic insulin concentration point has been defined as $1 /\left(\hat{y}_{k} C V_{I}\right)^{2}$, where $C V_{I}$ is the coefficient of variation for insulin. For each subject IRWLS parameter estimates of $\theta$ were obtained for several different values of the coefficients of variation $C V_{G}$ and $C V_{I}$, namely:

$$
\begin{aligned}
&\left(C V_{G}, C V_{I}\right) \in \quad\{(0.015,0.07),(0.02,0.10),(0.03,0.10),(0.03,0.15), \\
&(0.04,0.15),(0.05,0.15),(0.15,0.30)\} .
\end{aligned}
$$

These sets of coefficients of variation were used in order to conduct a sensitivity analysis on the diffusion coefficient (defined in section 2.4 below) by considering different values for the variance of the measurement error, which is assumed proportional to the square of the coefficient of variation.

The starting point to fix reasonable values for $C V_{G}$ and $C V_{I}$ was suggested in Bergman et al. (1979) where it had been found $\left(C V_{G}, C V_{I}\right)=(0.015,0.07)$; nevertheless, since these values refer to in vitro estimates of the variance of repeated laboratory measurements on the same preparation, it could be more realistic to re-estimate $C V_{G}$ and $C V_{I}$ from data. To this aim we adopted a General Least Squares approach (GLS (Davidian and Giltinan, 1995, chapter 5 ), detailed in appendix). 


\subsection{Stochastic model}

As an alternative to the above deterministic model, we may assume that the underlying tissue glucose uptake process is not smooth, subject as it is to a variety of metabolic and hormonal influences, which change over time. In fact, tissue glucose uptake is determined not only by the varying concentrations of certain hormones (e.g. cortisol or growth hormone) and by the rhythm of food intake, events which take place over periods of hours, but also by sudden changes in physical activity or emotional stresses induced by thought processes. We may thus imagine that the insulin-dependent glucose disposal rate may be subject to moment-bymoment variations and that the rate constant $K_{x g I}$ is likely to exhibit substantial irregular oscillations over time. Thus we define two sources of noise: a dynamic noise term, which is a part of the process, such that the value of the process at time $t$ depends on this noise up to time $t$, and a measurement noise term, which does not affect the process itself, but only its observations.

We therefore allow the parameter $K_{x g I}$ to vary randomly as $\left(K_{x g I}-\xi(t)\right)$, where $\xi(\cdot)$ is a gaussian white-noise process. Then the system noise $\xi(t) d t$ can be written as $\sigma d W(t)$ (see e.g. Ditlevsen and De Gaetano (2005), Kloeden and Platen (1992) and Øksendal (2000)), where $\sigma \geq 0$ represents the (unknown) diffusion coefficient and $W(\cdot)$ is the Wiener process (Brownian motion), which is a random process whose increments are independent and normally distributed with zero mean and with variance equal to the length of the time interval over which the increment take place. By incorporating the $K_{x g I}$ variation into the deterministic model, we obtain the following (Itô) SDE:

$$
\begin{aligned}
d G(t)= & {\left[\frac{\left(T_{g x}\left(t-\tau_{g}\right)+T_{g h}(t)\right)}{V_{g}}-T_{x g} \frac{G(t)}{0.1+G(t)}-K_{x g I} G(t) I(t)\right] d t } \\
& +\sigma G(t) I(t) d W(t) \\
d I(t)= & {\left[\frac{\left(T_{i G} G(t)+T_{i x}(t)\right)}{V_{i}}-K_{x i} I(t)\right] d t, } \\
T_{g h}(t)= & T_{g h \max } \exp (-\lambda G(t) I(t))
\end{aligned}
$$

with $G(0)=G_{b}, I(0)=I_{b}$ and $T_{g h}(0)=T_{g h b}=T_{g h m a x} \exp \left(-\lambda G_{b} I_{b}\right)$. Notice that this formulation has the theoretical advantage of never becoming negative in any of the coordinates.

\subsection{SDE estimation}

While the model estimation procedure for systems of ODEs is well established, estimating parameters in SDE models is not straightforward, except for simple cases. A variety of methods for statistical inference in discretely observed diffusion processes have been developed during the past decades (e.g. Aït-Sahalia (2002), Bibby et al. (2005), Bibby and Sørensen (1995), Dacunha-Castelle and Florens-Zmirnou (1986), Elerian et al. (2001), Gallant and Long (1997), Gouriéroux et al. (1993), Hurn et al. (2003), Pedersen (1995), Pedersen (2001), Shoji and Ozaki (1998), Sørensen (2000), Yoshida (1992)). A natural approach would be maximum likelihood inference, but it is rarely possible to write the likelihood function explicitly. In our case it becomes further complicated since we are dealing with partially observed state variables. The estimation approach we follow is to first estimate by IRWLS the parameters of the ODE system (1)-(3) (as explained in section 2.3), which represents the deterministic part (drift) of the SDE model and the mean of the corresponding stochastic process. We then use the MonteCarlo approximation to the unknown likelihood function as suggested in Pedersen (2001), in 
order to estimate $\sigma$ by keeping fixed the previously obtained drift parameter estimates. We make recourse to the method in Pedersen (2001) since plasma glucose concentrations $G$ and serum insulin concentrations $I$ are not observed at the same time-points (every $5 \mathrm{~min}$. and every $20 \mathrm{~min}$. respectively), and we therefore deal with partially observed state variables, and further because the concentrations are observed with measurement error. In the following the application of the method to the problem under investigation is detailed; for ease of notation we denote with $Z_{i}$ the generic variable $Z\left(t_{i}\right)$ at time $t_{i}$. Consider the model (4)-(6) and observation times $0=t_{1} \leq t_{2} \leq \cdots \leq t_{n}$ : all the observations $y$ are collected in a single array and distinguished using the following label-variable

$$
\chi_{i}=\left\{\begin{array}{ll}
G, & \text { if the observation at time } t_{i} \text { refers to glucose } \\
I, & \text { if the observation at time } t_{i} \text { refers to insulin }
\end{array} \quad i=1, \ldots, n\right.
$$

We consider the error-model

$$
Y_{i}=H_{\chi_{i}}+\varepsilon_{i}
$$

where

$$
H_{\chi_{i}}=\left\{\begin{array}{l}
G_{i}, \quad \text { if } \chi_{i}=G \\
I_{i}, \quad \text { if } \chi_{i}=I
\end{array} \quad i=1, \ldots, n\right.
$$

and the $\varepsilon_{i}$ 's are independent normal variables with mean 0 and variance $\sigma_{\chi_{i}}^{2}$ representing the measurement errors. We assume that (i)

$$
\sigma_{\chi_{i}}^{2}=\left\{\begin{array}{l}
\left(C V_{G} G_{i}\right)^{2}, \quad \text { if } \chi_{i}=G \\
\left(C V_{I} I_{i}\right)^{2}, \quad \text { if } \chi_{i}=I
\end{array} \quad i=1, \ldots, n\right.
$$

where $C V_{G}$ and $C V_{I}$ represent the coefficient of variations for the glucose and the insulin concentrations respectively, and that (ii) the measurement errors are independent of the process $W(\cdot)$. Equation (7) and the SDE system (4)-(6) provide a representation of the error-structure in our problem. Denote with $y_{i}$ the observed value of $Y_{i}$ at time $t_{i}$, then the likelihood function of $\sigma$ can be written as

$$
\begin{aligned}
L(\sigma) & =\int_{\mathbb{R}^{n-2}}\left[\prod_{i=1}^{n} g_{i}\left(y_{i} \mid H_{\chi_{i}} ; \sigma\right)\right] f\left(H_{\chi_{3}}, \ldots, H_{\chi_{n}} ; H_{\chi_{1}}, H_{\chi_{2}}, \sigma\right) d H_{\chi_{3}} \cdots d H_{\chi_{n}} \\
& =\mathbb{E}_{\sigma} \prod_{i=1}^{n} g_{i}\left(y_{i} \mid H_{\chi_{i}} ; \sigma\right)
\end{aligned}
$$

where $H_{\chi_{1}}=G_{b}$ and $H_{\chi_{2}}=I_{b}$ are the initial conditions of $G$ and $I$ respectively, $f$ denotes the (unknown) joint density function of $H_{\chi_{3}}, \ldots, H_{\chi_{n}}$ given $\left(H_{\chi_{1}}, H_{\chi_{2}}, \sigma\right), \mathbb{E}_{\sigma}$ denotes expectation w.r.t. the distribution of $H_{\chi_{3}}, \ldots, H_{\chi_{n}}$ for the indicated value of $\sigma$ and

$$
g_{i}\left(y_{i} \mid H_{\chi_{i}} ; \sigma\right)=\left(2 \pi \sigma_{\chi_{i}}^{2}\right)^{-1 / 2} \exp \left[-\frac{1}{2 \sigma_{\chi_{i}}^{2}}\left(y_{i}-H_{\chi_{i}}\right)^{2}\right]
$$

is the normal density function with expectation $H_{\chi_{i}}$ and variance $\sigma_{\chi_{i}}^{2}$. If $H^{r}(r=1, \ldots, R)$ are stochastically independent random vectors, each distributed as $\left(H_{\chi_{3}}, \ldots, H_{\chi_{n}}\right)$, then it follows from the strong law of large numbers that the likelihood function can, for large values of $R$, be approximated by

$$
L(\sigma) \simeq \frac{1}{R} \sum_{r=1}^{R} \prod_{i=1}^{n} g_{i}\left(y_{i} \mid H_{\chi_{i}}^{r} ; \sigma\right)
$$


In practice the approximation is obtained by simulating the $H_{\chi_{i}}^{r}$ 's (see Kloeden and Platen (1992)) for a large finite number $R$.

We have initially simulated $R=1000,2000$ and 4000 trajectories of the process according to the Euler-Maruyama scheme Kloeden and Platen (1992) with an integration step-size of 0.1 min.: given a $\sigma$ of the order of $10^{-5}$, the step-size ensures a standard deviation for $d G$ smaller than 0.02 in each integration step, which is very small compared to the order of magnitude of the glucose concentrations. The simulated likelihood functions did not appreciably change the location of their maximum when increasing the number of trajectories beyond 2000. Therefore the reported estimates of $\sigma$ were obtained by maximizing the approximated likelihood (8), based on $R=2000$ trajectories, when keeping fixed the parameters entering the drift part of the model and using different combinations of levels of $C V_{G}$ and $C V_{I}$ (see section 2.3), in order to explore the sensitivity of the obtained estimates $\hat{\sigma}$ to mis-specification of the observation error.

\section{Results}

\subsection{Deterministic differential model}

We first estimated by IRWLS, separately on each subject, the structural (free) parameters $G_{b}$, $I_{b}, K_{x g I}, K_{x i}, T_{g h \max }, V_{g}, V_{i}, \tau_{g}, \lambda$, and computed the corresponding structural (determined) parameters $T_{g h b}, T_{x g}$ and $T_{i G}$ entering the deterministic model (1)-(3): the estimates corresponding to three different choices for the coefficients of variation (see section 2.3) are reported in tables 4,5 and 6, whereas graphical results of the IRWLS fitting are shown in figures 2 and 3 only for the case $\left(C V_{G}, C V_{I}\right)=(0.05,0.15)$ (in fact these values are very similar to the GLS population-estimates of $C V_{G}$ and $C V_{I}$, as explained below), since in our problem the predicted curves do not vary substantially with different measurement error values.

Secondarily, we estimated the individual structural parameters, as well as the population parameters $C V_{G}$ and $C V_{I}$, simultaneously by GLS (Table 7): in this way we found that $\widehat{C V}_{G}=0.071$ and $\widehat{C V}_{I}=0.1702$.

\subsection{Stochastic differential model}

The stochastic model (4)-(6) was adapted to our data and $\sigma$ was estimated as described in section 2.5. The estimates of $\sigma$ corresponding to the different sets of coefficients of variation are reported in Table 8 for each subject. In this table we notice that the $\sigma$ estimates are stable when considered in a reasonable region of the coefficient of variations values, that is when considered in $\left(C V_{G}, C V_{I}\right) \in[0.02,0.05] \times[0.10,0.15]$. At the smallest level $\left(C V_{G}, C V_{I}\right)=$ $(0.015,0.07)$ the $\sigma$ estimates result numerically unidentifiable for three subjects, and are thus marked with an 'NA'.

While theoretically we could have tried to estimate simultaneously all parameters appearing in the SDE model (drift parameters, diffusion coefficient determining the system noise variance, observation error variance), from a purely computational point of view this proved exceedingly expensive and we had to be content with a sequential estimation approach. In this way, several combinations of observation error levels were hypothesized, the drift parameters were estimated under each hypothesis, and the corresponding diffusion was then estimated in each case. 
For illustration purposes, graphical results of the fitting only for the cases $\left(C V_{G}, C V_{I}\right)=$ $(0.05,0.15)$ and $\left(C V_{G}, C V_{I}\right)=(0.03,0.15)$ are shown in figures 4 and 5 respectively, only for the glycemia values since the insulin curves are almost identical to those produced by the deterministic model. For each subject figures 4 and 5 report the observed glycemias and the empirical mean of $R=2000$ simulated trajectories of the $G(t)$ process, their empirical $95 \%$ confidence limits (from the 2.5 th percentile to the 97.5 th percentile) and one simulated trajectory. According to Pedersen (1994)-Pedersen (2001) we are able to check the plausibility of our stochastic model by simulating uniform residuals; the q-q plots of the simulated uniform residuals are reported in figures 6 and 7 , where the residuals are plotted against percentiles from the $U(0,1)$ distribution. The caption of each subfigure also reports the $p$-value from the two-tailed Kolmogorov-Smirnov goodness-of-fit test; if $p<0.05$ the simulated residuals do not conform to the hypothesis of $U(0,1)$ distribution at a $5 \%$ confidence level. The tests have not been subjected to correction for simultaneous inference (Bonferroni or similar) in order to be more conservative. All tests had $p>0.05$, except for the glycemia residuals for subjects 4 and 6 and for the insulinemia residuals for subjects 8 and 15.

\section{Discussion}

The Euglycemic Hyperinsulinemic Clamp is the procedure most commonly employed by research diabetologists in their quest for the determination of the degree of insulin sensitivity (or resistance) exhibited by a given experimental subject. In its common usage, after having "clamped", i.e. stabilized, the subject's glycemia to pre-insulinization levels, the average rate of glucose infusion necessary to maintain euglycemia is measured, and is directly employed (once normalized by the subject's body mass) as an index of insulin sensitivity.

As an alternative to the above, a model may be drawn to describe the mass flow of glucose into and out the central (sampling) plasma compartment of the subject, explicitly representing the physiological mechanisms known to intervene in the process.

In the present work, a (simple) deterministic model of the clamp procedure is studied first. The main result of this study is that the level of error around the predicted curve is very large, in particular it is much larger than the $(0.015,0.07)$ commonly accepted levels of measurement error in in vitro repeated testing of the same laboratory preparation. This result would theoretically be compatible with either one of the following alternatives: the model is misspecified; the in vivo measurement error is in reality much larger than $(0.015,0.07)$; or, there is some additional source of noise, besides measurement error, which substantially impacts observations.

From an examination of Figure 2, it would not seem that the average model prediction is systematically wrong. Similarly, the coefficient of variations estimated by GLS around the deterministic prediction are much too large to be compatible with measurement error. The idea that glucose absorption by tissues varies in time is, on the other hand, rather natural: it seems evident that, subject to variable hormonal concentrations, variable stress levels, even to minor posturali changes, muscle uptake and liver output of glucose may vary from moment to moment. What remains to be seen is if a mathematical model incorporating this idea would be supported by the actual observations.

A random fluctuation in the net tissue glucose uptake rate is a reasonable approximation to 
the effect of a host of the poorly controlled, additive influences mentioned. When considering this random fluctuations as well, the original deterministic model (1)-(3) is thus transformed into the SDE model (4)-(6).

The approach followed for the estimation of the relevant quantities of the model (structural parameters and diffusion) is motivated by the computing-intensive algorithms necessary for the estimation of the diffusion, which require the simulation of thousands of possible trajectories of the process for every evaluation of the merit function.

Essentially, it has been shown that: (i) for any reasonable level of observation error, the estimated diffusion has more or less the same value. For "reasonable" it is here meant larger than pure measurement error and smaller than the total error around the expected trajectory as estimated by GLS. Adopting the lowermost observation error level $(0.015,0.07)$ would be equivalent to stating that the same variability exists on repeated laboratory measurements on the same sample as on repeated sampling/measurement procedures at the same actual glycemia and insulinemia, disregarding further potential sources of variation accruing to the sampling procedure itself (volume of blood vs. volume of anticoagulant, degree of coagulation, variation in spinning time etc.): this seems extreme in one direction. In the other direction, the largest error level considered $(0.15,0.30)$ is much higher than the total observation variability actually estimated with the GLS procedure around the deterministic prediction $(0.071,0.1702)$, and for this reason should also be discarded. Having excluded these extreme cases, it can be seen that, in the present situation, the estimation of the diffusion is very robust to changes in the likely value of the observation error, as similarly robust are the estimates of the structural (drift) parameters. Further (ii) we showed that the diffusion coefficient estimates are generally strictly positive: this means that the dynamical process which most likely represents the glycemia time-course (given the estimated deterministic differential model) is a stochastic process with a non-negligible system noise, whose intensity factor is represented by the diffusion coefficient. Pictorial evidence of the magnitude of diffusion is given in figures 4 and 5 . This system noise represents the additive action of many factors, each with a small individual effect, which are not explicitly represented in the deterministic model (that is in the drift term of the SDE), and which instantaneously affect glucose uptake rate. Therefore, in the stochastic differential model the collective influence of many individually neglected effects is added to the average drift term, which, on the other side, represents the most relevant and generally well-recognized factors affecting glycemia.

It is interesting to note from figures 4 and 5 that when the process average (which also represents the ordinary differential model solution) fits well the observed glycemias, its $95 \%$ confidence band is narrow (e.g. subjects 1, 2, 3, 10 and 12). On the other hand, when the process average itself is not able to meaningfully capture the general trend of the observations, the corresponding confidence band is much larger (e.g. subjects 4, 5 and 9). In these last cases, the system is driven more by the erratic oscillations in tissue glucose transport rather than by the smooth dynamics of the overall actual system, of course under the hypothesis that the proposed deterministic term is correctly specified. This finding would prompt us to reconsider the quantitative relevance of the unexpressed effects, in other situations, represented so far only with deterministic models, especially when less than perfectly satisfactory fits to data have been obtained.

The particular behavior of the estimated diffusion (Table 8), for the different choices of coefficient of variation values $\xi \equiv\left(C V_{G}, C V_{I}\right)$ could seem counterintuitive: one would expect 
that as the observation error is assumed to increase, the estimated system error should decrease (this actually happens for macroscopically exaggerated values of the observation error). This unexpected result may be due to the estimation method we adopted: since the array of free structural parameters $\theta$ for the drift part of the stochastic model is estimated anew for different values of $\xi$, the $\hat{\theta}$ 's are depending on these levels of error, so we can write $\hat{\theta} \equiv \hat{\theta}_{\xi}$. As a consequence of that, the (numerical) solution of the SDE system (4)-(6) and the measurement error $\varepsilon$ are not independent and, by means of equation (7), we can write

$$
\begin{aligned}
\operatorname{Var}\left(Y_{i}\right) & =\operatorname{Var}\left(H_{\chi_{i}}\left(\hat{\theta}_{\xi}\right)\right)+\operatorname{Var}\left(\varepsilon_{i}\right)+2 \operatorname{Cov}\left(H_{\chi_{i}}\left(\hat{\theta}_{\xi}\right), \varepsilon_{i}\right) \\
& =\operatorname{Var}\left(H_{\chi_{i}}\left(\hat{\theta}_{\xi}\right)\right)+\sigma_{\chi_{i}}^{2}+2 \mathbb{E}\left(H_{\chi_{i}}\left(\hat{\theta}_{\xi}\right) \varepsilon_{i}\right) .
\end{aligned}
$$

Since $H(\cdot)$ is unknown, so is $\mathbb{E}\left(H_{\chi_{i}}\left(\hat{\theta}_{\xi}\right) \varepsilon_{i}\right)$ and we cannot compute the covariance analytically: however it is generally not zero, and the variance of the observations is not the simple sum of the variance of the trajectories and of the variance of the measurement error.

There is indeed the possibility that the parameters of the system, or the model structure itself, may be non-stationary over the time course of the experiment. If this non-stationarity is judged to be potentially important, it can be represented by actually modeling the time-course of the parameter value over the duration of the experiment, as a function of other (meta-) parameters. The statistical significance of such meta-parameters would then indicate whether the influence of this non-stationarity is relevant. Similarly for a possible variation of the model structure over time.

It is of interest to note that even when glycemia is allowed to vary stochastically, responding to the Wiener process introduced in equation (4), the corresponding oscillations in insulinemia are very small, and the insulin process does not substantially differ from its own expected value. This may very well be explained when considering the relative inertia of the pancreatic insulin secretion mechanism, coupled with the large volume of distribution of the hormone, which combine to minimize oscillations in insulin concentrations in response to rapidly varying glucose concentrations.

We conclude therefore that the stochastic differential model (4)-(6) is statistically robust, physiologically meaningful and represents well the glucose metabolism occurring during a clamp study. More generally, it can be concluded that stochastic differential equations are theoretically useful and practically applicable, and deserve to be considered more often as a valuable addition to the biomedical modeller's toolbox.

Acknowledgements: The authors are grateful to Prof. G. Mingrone (Università Cattolica del Sacro Cuore, Policlinico Universitario "A. Gemelli", Rome, Italy) for having provided the original data sets and commented on the EHC procedure. The work was supported by grants from the Danish Medical Research Council and the Lundbeck Foundation to S. Ditlevsen.

\section{$5 \quad$ Appendix}

To obtain subject-specific regression parameters and population estimates of $C V_{G}$ and $C V_{I}$ the GLS method was performed (Davidian and Giltinan, 1995, chapter 5). The GLS is a 
two-stage method: (stage 1) at first individual estimates for each subject $i(i=1, \ldots, 15)$ were obtained; then (stage 2) these estimates were used as building blocks to construct the population estimates of $C V_{G}$ and $C V_{I}$.

Suppose that $y_{i}$ and $\theta_{i}$ represent the $n_{i}$-dimensional array of recorded data and the array of (structural) individual parameters for subject $i$ respectively $(i=1, \ldots, 15)$, i.e. $\theta_{i}$ contains the values of the (free) parameters $\theta=\left(G_{b}, I_{b}, K_{x g I}, K_{x i}, T_{g h m a x}, V_{g}, V_{i}, \tau_{g}, \lambda\right)$ entering the model (1)-(3) for subject $i$. Consider now the model

$$
y_{i}=f_{i}\left(\theta_{i}\right)+\varepsilon_{i}
$$

such that

$$
\mathbb{E}\left(\varepsilon_{i} \mid \theta_{i}\right)=0, \quad \operatorname{Cov}\left(\varepsilon_{i} \mid \theta_{i}\right)=\Omega_{i}\left(\theta_{i}, \xi\right)
$$

with $f_{i}(\cdot)$ representing the numerical solution of the system (1)-(3) for subject $i$, and assuming that the functional form of $\Omega_{i}(\cdot, \cdot)$ and the intra-individual covariance parameter $\xi=\left(C V_{G}, C V_{I}\right)$ are the same across individuals. If we denote with $G$ and $I$ the state variable Glucose and Insulin respectively, the covariance matrix $\Omega_{i}\left(\theta_{i}, \xi\right)$ in the present application has the structure of an $n_{i} \times n_{i}$ block-diagonal matrix

$$
\Omega_{i}\left(\theta_{i}, \xi\right)=\left(\begin{array}{cc}
\Omega_{i, G} & 0 \\
0 & \Omega_{i, I}
\end{array}\right) \quad i=1, \ldots, 15
$$

where

$$
\begin{aligned}
\Omega_{i, G} & =\left(\begin{array}{cccc}
C V_{G}^{2} f_{i G}^{2}\left(\theta_{i}, t_{i, 1}\right) & 0 & \ldots & 0 \\
\cdots & \ldots & \ldots & \ldots \\
0 & \cdots & 0 & C V_{G}^{2} f_{i G}^{2}\left(\theta_{i}, t_{i, n_{i G}}\right)
\end{array}\right), \\
\Omega_{i, I} & =\left(\begin{array}{cccc}
C V_{I}^{2} f_{i I}^{2}\left(\theta_{i}, t_{i, 1}\right) & 0 & \ldots & 0 \\
\cdots & \ldots & \ldots & \ldots \\
0 & \cdots & 0 & C V_{I}^{2} f_{i I}^{2}\left(\theta_{i}, t_{i, n_{i I}}\right)
\end{array}\right)
\end{aligned}
$$

with $f_{i G}\left(\theta_{i}, t_{i, j_{1}}\right)$ and $f_{i I}\left(\theta_{i}, t_{i, j_{2}}\right)$ representing the predicted glycemia and insulinemia values at times $t_{i, j 1}$ and $t_{i, j 2}$ respectively $\left(j_{1}=1, \ldots, n_{i G} ; j_{2}=1, \ldots, n_{i I} ; n_{i G}+n_{i I}=n_{i}\right)$. Then the GLS algorithm is given by the following scheme:

1. in $m=15$ separate regressions, obtain preliminary estimates $\hat{\theta}_{i}^{(p)}$ for each individual, $i=1, \ldots, m$;

2. use residuals from these preliminary fits to estimate $\xi$ by minimizing the following functional

$$
\sum_{i=1}^{m} P L_{i}\left(\hat{\theta}_{i}^{(p)}, \xi\right)=\sum_{i=1}^{m} \log \left|\Omega_{i}\left(\hat{\theta}_{i}^{(p)}, \xi\right)\right|+\left(y_{i}-f_{i}\left(\hat{\theta}_{i}^{(p)}\right)\right)^{\prime} \Omega_{i}^{-1}\left(\hat{\theta}_{i}^{(p)}, \xi\right)\left(y_{i}-f_{i}\left(\hat{\theta}_{i}^{(p)}\right)\right)
$$

where $P L_{i}$ is the pseudolikelihood of $\xi$ for the $i$ th individual. Form estimated weight matrices based on the estimate $\hat{\xi}$ obtained from this procedure, along with the preliminary $\hat{\theta}_{i}^{(p)}$, to form

$$
\hat{\Omega}_{i}\left(\hat{\theta}_{i}^{(p)}, \hat{\xi}\right)
$$


3. using the estimated weight matrices from step 2 , re-estimate the $\theta_{i}$ 's by $m$ separate minimizations: for individual $i$, minimize in $\theta_{i}$

$$
\left(y_{i}-f_{i}\left(\theta_{i}\right)\right)^{\prime} \hat{\Omega}_{i}^{-1}\left(y_{i}-f_{i}\left(\theta_{i}\right)\right)
$$

Treating the resulting estimators as new preliminary estimators, return to step 2.

The algorithm should be iterated at least once to eliminate the effect of potentially inefficient preliminary estimates in step 1 .

\section{References}

Aït-Sahalia, Y. (2002). Maximum likelihood estimation of discretely sampled diffusions: a closed-form approximation approach. Econometrica, 70(1), 223-262.

Bergman, R., Ider, Y., Bowden, C., and Cobelli, C. (1979). Quantitative estimation of insulin sensitivity. Am. J. Physiol., 236, E667-E677.

Bibby, B. and Sørensen, M. (1995). Martingale estimation functions for discretely observed diffusion processes. Bernoulli, 1(1/2), 17-39.

Bibby, B., Jacobsen, M., and Sørensen, M. (2005). Estimating functions for discretely sampled diffusion-type models. In Y. Aït-Sahalia and L. Hansen, editors, Handbook of Financial Econometrics. Amsterdam: North-Holland.

Dacunha-Castelle, D. and Florens-Zmirnou, D. (1986). Estimation of the coefficients of a diffusion from discrete observations. Stochastics, 19, 263-284.

Davidian, M. and Giltinan, D. (1995). Nonlinear models for repeated measurement data. Chapman \& Hall.

DeFronzo, R. and Ferrannini, E. (1991). Insulin resistance. a multifaceted syndrome responsible for NIDDM, obesity, hypertension, dyslipidemia, and atherosclerotic cardiovascular disease. Diabetes Care, 14, 173-194.

DeFronzo, R., Tobin, J., and Andres, R. (1979). Glucose clamp technique: a method for quantifying insulin secretion and resistance. Am. J. Physiol., 237, E214-E223.

Ditlevsen, S. and De Gaetano, A. (2005). Stochastic vs. deterministic uptake of dodecanedioic acid by isolated rat livers. Bull. Math. Biol., 67, 547-561.

Elerian, O., Shephard, N., and Chib, S. (2001). Likelihood inference for discretely observed nonlinear diffusions. Econometrica, 69, 959-993.

Ferrannini, E. and Mari, A. (1998). How to measure insulin sensitivity. J. Hypertens., 16, 895-906.

Gallant, A. and Long, J. (1997). Estimating stochastic differential equations efficiently by minimum chi-square. Biometrika, 84, 124-141.

Gouriéroux, C., Monfort, A., and Renault, E. (1993). Indirect inference. Journal of Applied Econometrics, 8, 85-118. 
Hurn, A., Lindsay, K., and Martin, V. (2003). On the efficacy of simulated maximum likelihood for estimating the parameters of stochastic differential equations. Journal of Time Series Analysis, 24(1), 45-63.

Kloeden, P. E. and Platen, E. (1992). Numerical solution of stochastic differential equations. Springer.

Øksendal, B. (2000). Stochastic differential equations. Springer, second edition.

Olson, A. and Pessin, J. (1996). Structure, function, and regulation of the mammalian facilitative glucose transporter gene family. Annu. Rev. Nutr., 16, 235-256.

Pedersen, A. R. (1994). Uniform residuals for discretely observed diffusion processes. Technical Report 292, Department of Theoretical Statistics, University of Aarhus, Denmark.

Pedersen, A. R. (1995). A new approach to maximum likelihood estimation for stochastic differential equations based on discrete observations. Scand. J. Statist., 22(1), 55-71.

Pedersen, A. R. (2001). Likelihood inference by monte carlo methods for incompletely discretely observed diffusion processes. Technical Report 1, Department of Biostatistics, University of Aarhus, Denmark.

Picchini, U., De Gaetano, A., Panunzi, S., Ditlevsen, S., and Mingrone, G. (2005). A mathematical model of the euglycemic hyperinsulinemic clamp. Theor. Biol. Med. Model., 2(44).

Sacks, W. (1969). In A. Lajtha, editor, Handbook of Neurochemistry, volume 1. New York: Plenum.

Shoji, I. and Ozaki, T. (1998). Estimation for nonlinear stochastic differential equations by a local linearization method. Stochastic Analysis and Applications, 16, 733-752.

Sørensen, M. (2000). Prediction-based estimating functions. Econometrics Journal, 3, 123147.

Starke, A. (1992). Determination of insulin sensitivity: methodological considerations. $J$. Cardiovasc. Pharmacol., 20, S17-S21.

Tornøe, C., Jacobsen, J., and Madsen, H. (2004). Grey-box pharmacokinetic/pharmacodynamic modelling of a euglycaemic clamp study. J. Math. Biol., 48, 591-604.

Wallace, T. and Matthews, D. (2002). The assessment of insulin resistance in man. Diabet. Med., 19, 527-534.

Yoshida, N. (1992). Estimation of diffusion processes from discrete observations. Journal of Multivariate Analysis, 41, 220-242.

Zierler, K. (1999). Whole body glucose metabolism. Am. J. Physiol., 276, E409-E426. 
Table 1: Anthropometric and metabolic characteristics for the normal and obese $\left(^{*}\right)$ subjects; $B S A$ is the Body Surface Area $\left[\mathrm{m}^{2}\right]$ calculated via the DuBois formula $\left(B S A=0.20247 \cdot\right.$ height ${ }^{0.725}[\mathrm{~m}] \cdot$ weight $\left.{ }^{0.425}[\mathrm{~kg}]\right)$.

\begin{tabular}{lccccc}
\hline Subject & $B M I\left[\mathrm{~kg} / \mathrm{m}^{2}\right]$ & $B S A\left[\mathrm{~m}^{2}\right]$ & $G_{\text {fast }}[\mathrm{mM}]$ & $I_{\text {fast }}[\mathrm{pM}]$ & $I_{\text {max }}[\mathrm{pM}]$ \\
\hline 1 & 20.20 & 1.60 & 3.61 & 36.84 & 472.00 \\
$2^{*}$ & 35.93 & 2.38 & 4.83 & 108.42 & 607.30 \\
$3^{*}$ & 27.77 & 2.04 & 5.39 & 79.23 & 683.18 \\
$4^{*}$ & 38.10 & 2.11 & 5.11 & 139.00 & 625.76 \\
5 & 20.03 & 1.49 & 4.83 & 15.29 & 506.89 \\
6 & 19.33 & 1.55 & 3.67 & 21.55 & 464.54 \\
$7^{*}$ & 48.07 & 2.16 & 5.39 & 139.00 & 592.02 \\
8 & 18.51 & 1.55 & 4.06 & 13.90 & 527.00 \\
$9^{*}$ & 63.57 & 2.08 & 5.94 & 152.90 & 522.25 \\
10 & 18.59 & 1.46 & 5.44 & 49.34 & 482.42 \\
$11^{*}$ & 42.19 & 2.02 & 5.28 & 139.00 & 497.31 \\
12 & 22.59 & 1.71 & 3.39 & 32.66 & 469.22 \\
$13^{*}$ & 31.35 & 2.11 & 4.94 & 79.23 & 605.22 \\
$14^{*}$ & 27.91 & 1.83 & 4.61 & 83.40 & 679.62 \\
15 & 22.68 & 1.73 & 3.50 & 27.80 & 482.14 \\
\hline
\end{tabular}

Table 2: Definitions of the state variables.

\begin{tabular}{ll}
\hline Variables & time from insulin infusion start \\
\hline$t[\mathrm{~min}]$ & plasma glucose concentration at time $t$ \\
$G(t)[\mathrm{mM}]$ & serum insulin concentration at time $t$ \\
$I(t)[\mathrm{pM}]$ & glucose infusion rate at time $t$ \\
$T_{g x}(t)[\mathrm{mmol} / \mathrm{min} / \mathrm{kgBW}]$ & insulin infusion rate at time $t$ \\
$T_{i x}(t)[\mathrm{pmol} / \mathrm{min} / \mathrm{kgBW}]$ & net Hepatic Glucose Output (HGO) at time $t$ \\
$T_{g h}(t)[\mathrm{mmol} / \mathrm{min} / \mathrm{kgBW}]$ & \\
\hline
\end{tabular}




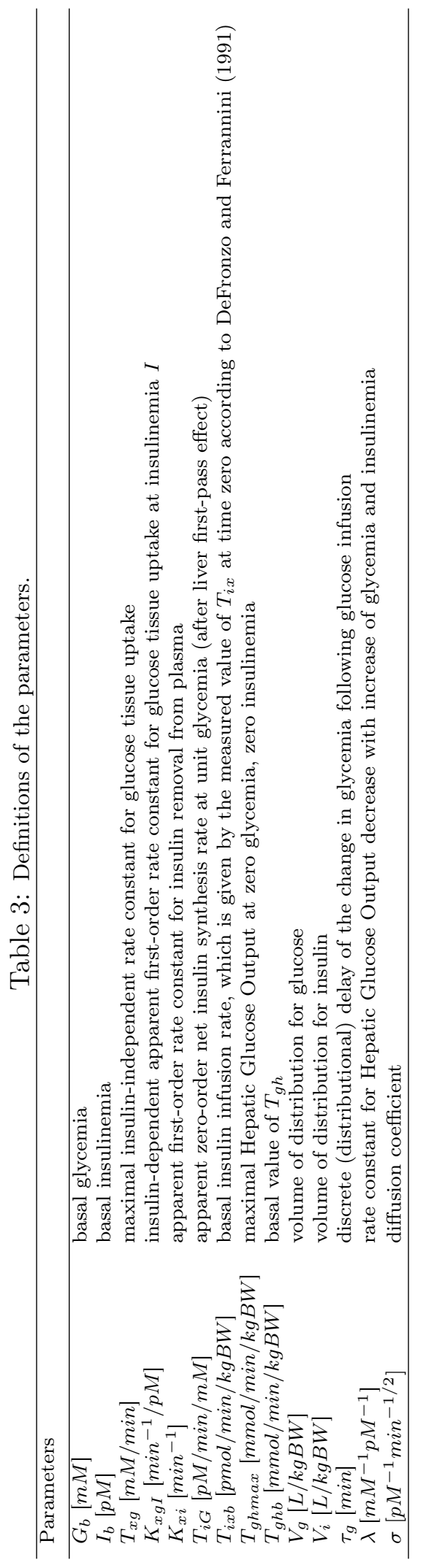




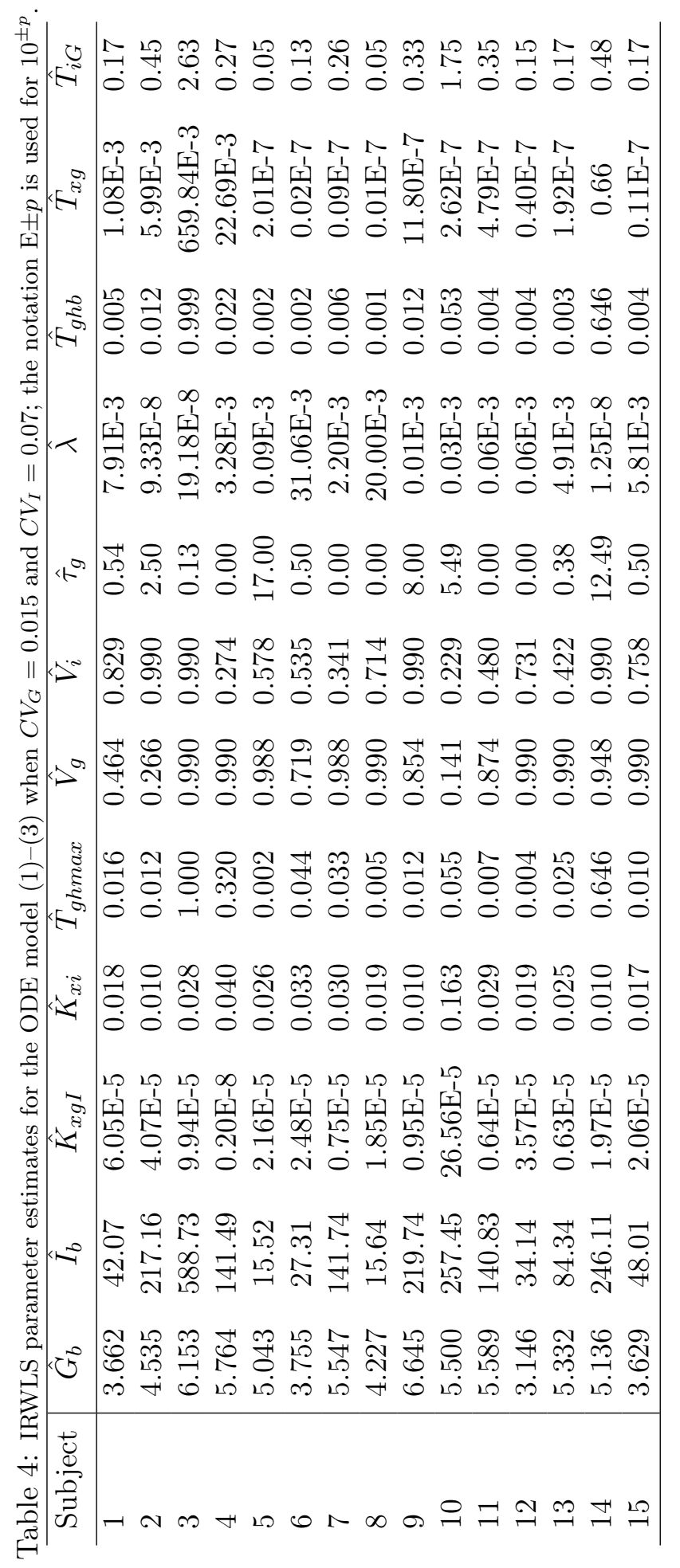




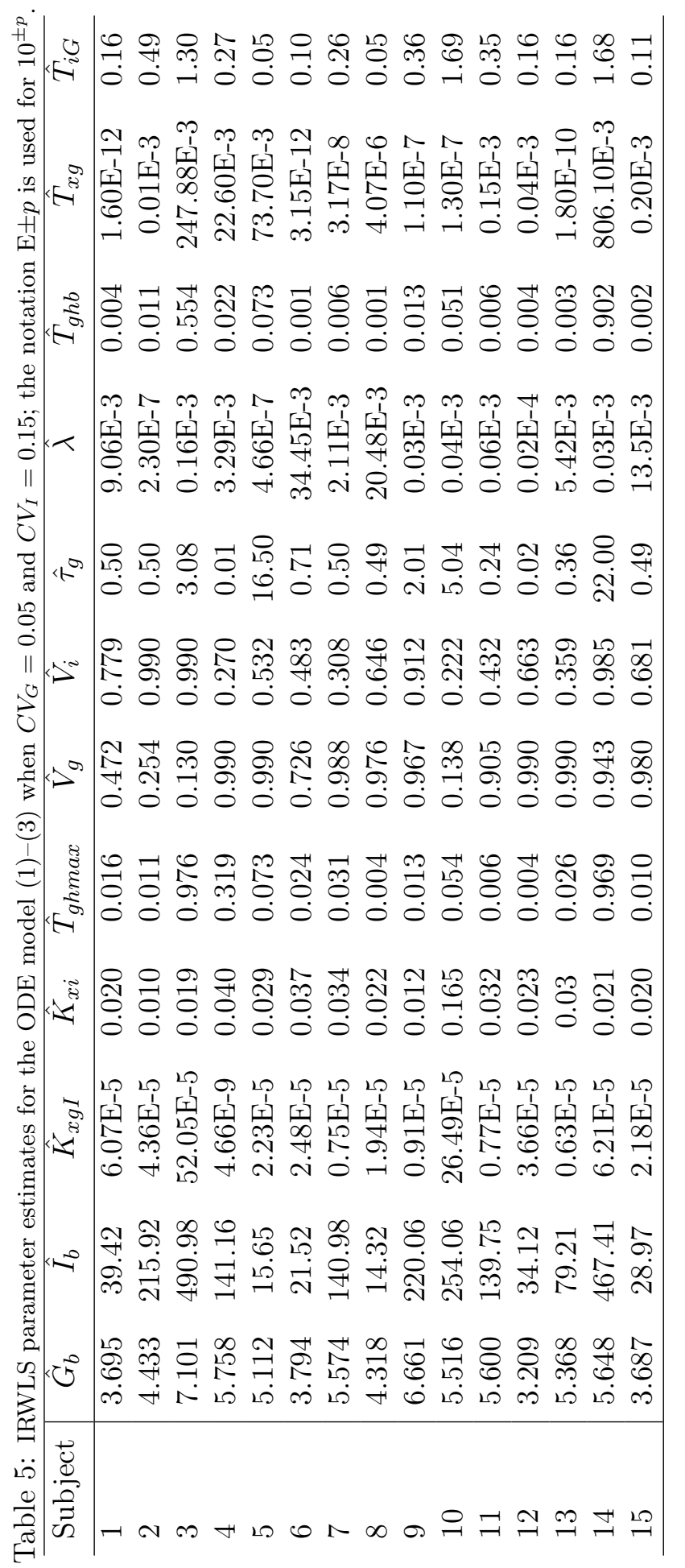




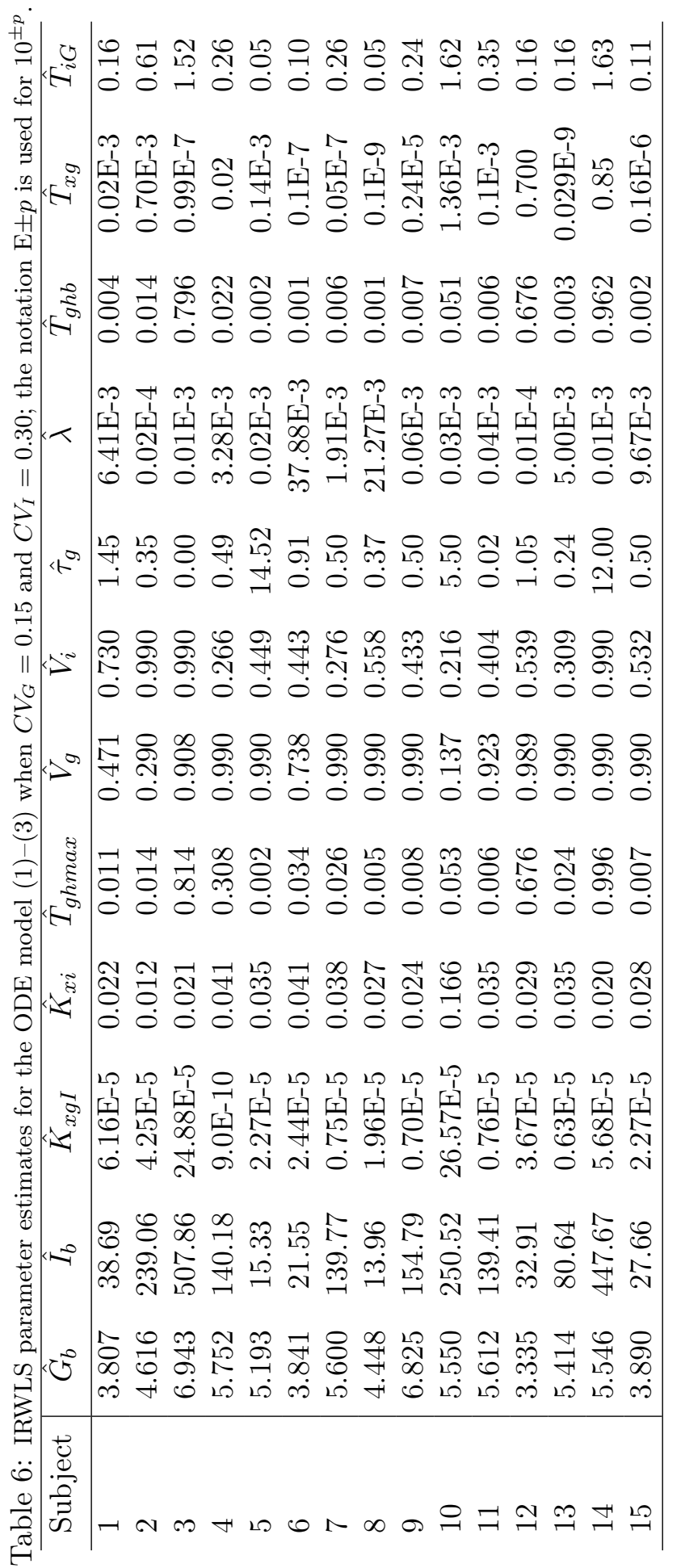




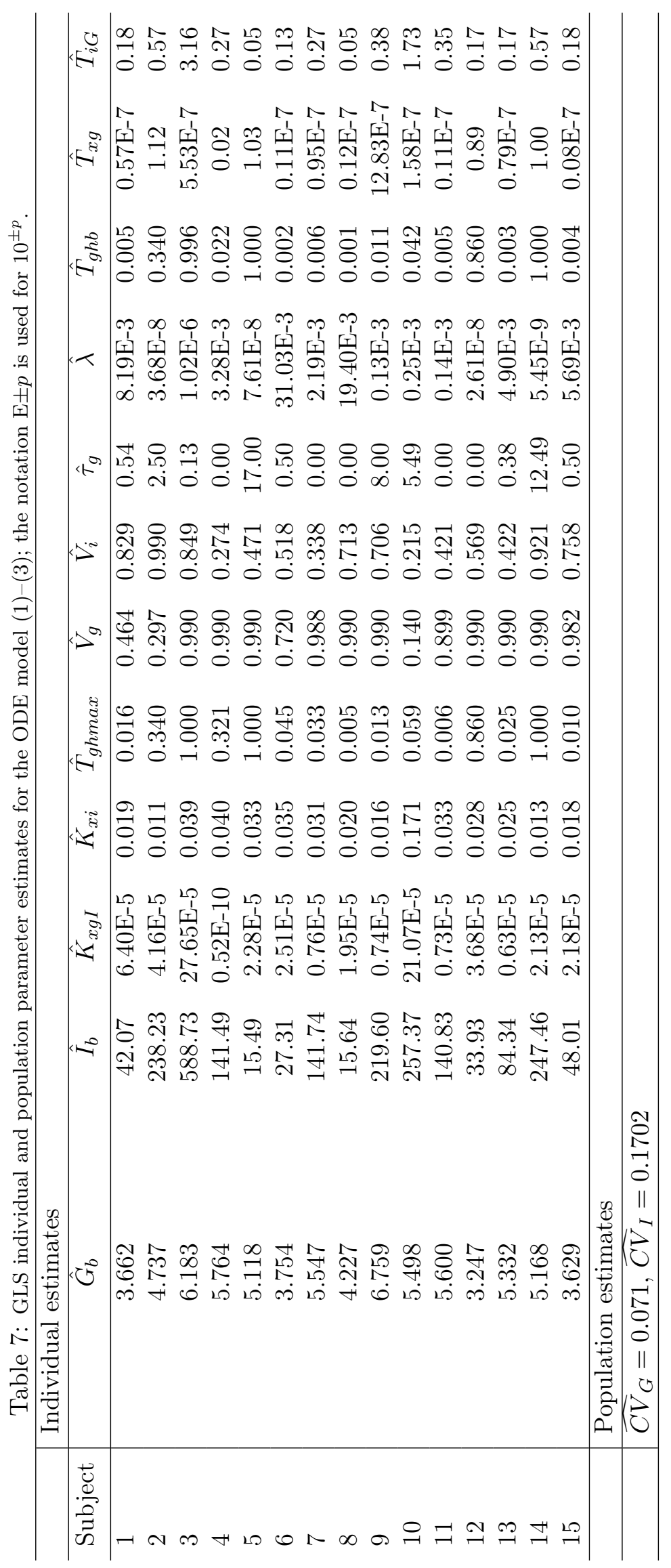


Table 8: Estimates of $\sigma$ in the cases $\left(C V_{G}, C V_{I}\right)=(0.015,0.07),\left(C V_{G}, C V_{I}\right)=(0.02,0.10),\left(C V_{G}, C V_{I}\right)=$ $(0.03,0.10), \quad\left(C V_{G}, C V_{I}\right)=(0.03,0.15),\left(C V_{G}, C V_{I}\right)=(0.04,0.15),\left(C V_{G}, C V_{I}\right)=(0.05,0.15)$ and $\left(C V_{G}, C V_{I}\right)=(0.15,0.30)$ given by $\hat{\sigma}^{(1)}, \hat{\sigma}^{(2)}, \hat{\sigma}^{(3)}, \hat{\sigma}^{(4)}, \hat{\sigma}^{(5)}, \hat{\sigma}^{(6)}$ and $\hat{\sigma}^{(7)}$ respectively. The notation $\mathrm{E} \pm p$ is used for $10^{ \pm p}$.

\begin{tabular}{l|ccccccc}
\hline Subjects & $\hat{\sigma}^{(1)}$ & $\hat{\sigma}^{(2)}$ & $\hat{\sigma}^{(3)}$ & $\hat{\sigma}^{(4)}$ & $\hat{\sigma}^{(5)}$ & $\hat{\sigma}^{(6)}$ & $\hat{\sigma}^{(7)}$ \\
\hline 1 & $1.60 \mathrm{E}-5$ & $1.78 \mathrm{E}-5$ & $2.25 \mathrm{E}-5$ & $1.59 \mathrm{E}-5$ & $2.10 \mathrm{E}-5$ & $2.25 \mathrm{E}-5$ & 0 \\
2 & NA & $1.38 \mathrm{E}-5$ & $1.47 \mathrm{E}-5$ & $1.38 \mathrm{E}-5$ & $1.38 \mathrm{E}-5$ & $1.15 \mathrm{E}-5$ & $2.88 \mathrm{E}-7$ \\
3 & $2.39 \mathrm{E}-5$ & $4.55 \mathrm{E}-5$ & $5.71 \mathrm{E}-5$ & $2.54 \mathrm{E}-5$ & $3.95 \mathrm{E}-5$ & $2.58 \mathrm{E}-5$ & 0 \\
4 & NA & $1.00 \mathrm{E}-5$ & $1.00 \mathrm{E}-5$ & $1.00 \mathrm{E}-5$ & $1.00 \mathrm{E}-5$ & $0.95 \mathrm{E}-5$ & $3.68 \mathrm{E}-8$ \\
5 & $1.83 \mathrm{E}-5$ & $1.97 \mathrm{E}-5$ & $2.00 \mathrm{E}-5$ & $1.93 \mathrm{E}-5$ & $1.93 \mathrm{E}-5$ & $1.93 \mathrm{E}-5$ & $0.91 \mathrm{E}-5$ \\
6 & $2.72 \mathrm{E}-5$ & $2.65 \mathrm{E}-5$ & $2.68 \mathrm{E}-5$ & $2.71 \mathrm{E}-5$ & $2.72 \mathrm{E}-5$ & $2.73 \mathrm{E}-5$ & $5.29 \mathrm{E}-8$ \\
7 & $0.80 \mathrm{E}-5$ & $0.80 \mathrm{E}-5$ & $0.80 \mathrm{E}-5$ & $0.80 \mathrm{E}-5$ & $2.35 \mathrm{E}-8$ & $2.35 \mathrm{E}-8$ & $1.47 \mathrm{E}-7$ \\
8 & $0.72 \mathrm{E}-5$ & $0.76 \mathrm{E}-5$ & $0.73 \mathrm{E}-5$ & $0.72 \mathrm{E}-5$ & $0.60 \mathrm{E}-5$ & $0.42 \mathrm{E}-5$ & $2.12 \mathrm{E}-7$ \\
9 & NA & $2.42 \mathrm{E}-5$ & $2.65 \mathrm{E}-5$ & $2.50 \mathrm{E}-5$ & $2.60 \mathrm{E}-5$ & $2.69 \mathrm{E}-5$ & $4.77 \mathrm{E}-7$ \\
10 & $3.08 \mathrm{E}-5$ & $3.04 \mathrm{E}-5$ & $3.04 \mathrm{E}-5$ & $3.04 \mathrm{E}-5$ & $3.00 \mathrm{E}-5$ & $2.88 \mathrm{E}-5$ & $4.25 \mathrm{E}-7$ \\
11 & $0.62 \mathrm{E}-5$ & $0.59 \mathrm{E}-5$ & $3.68 \mathrm{E}-8$ & $3.68 \mathrm{E}-8$ & $2.35 \mathrm{E}-8$ & $3.68 \mathrm{E}-8$ & $4.77 \mathrm{E}-7$ \\
12 & $1.44 \mathrm{E}-5$ & $0.98 \mathrm{E}-5$ & $1.36 \mathrm{E}-5$ & $1.41 \mathrm{E}-5$ & $1.47 \mathrm{E}-5$ & $1.53 \mathrm{E}-5$ & $8.47 \mathrm{E}-7$ \\
13 & $1.23 \mathrm{E}-5$ & $0.82 \mathrm{E}-5$ & $0.86 \mathrm{E}-5$ & $0.74 \mathrm{E}-5$ & $0.84 \mathrm{E}-5$ & $0.87 \mathrm{E}-5$ & $2.12 \mathrm{E}-7$ \\
14 & $1.73 \mathrm{E}-5$ & $1.65 \mathrm{E}-5$ & $1.64 \mathrm{E}-5$ & $1.62 \mathrm{E}-5$ & $1.56 \mathrm{E}-5$ & $0.12 \mathrm{E}-5$ & $7.21 \mathrm{E}-8$ \\
15 & $1.87 \mathrm{E}-5$ & $1.23 \mathrm{E}-5$ & $1.44 \mathrm{E}-5$ & $1.86 \mathrm{E}-5$ & $1.47 \mathrm{E}-5$ & $1.50 \mathrm{E}-5$ & $0.84 \mathrm{E}-5$ \\
\hline
\end{tabular}




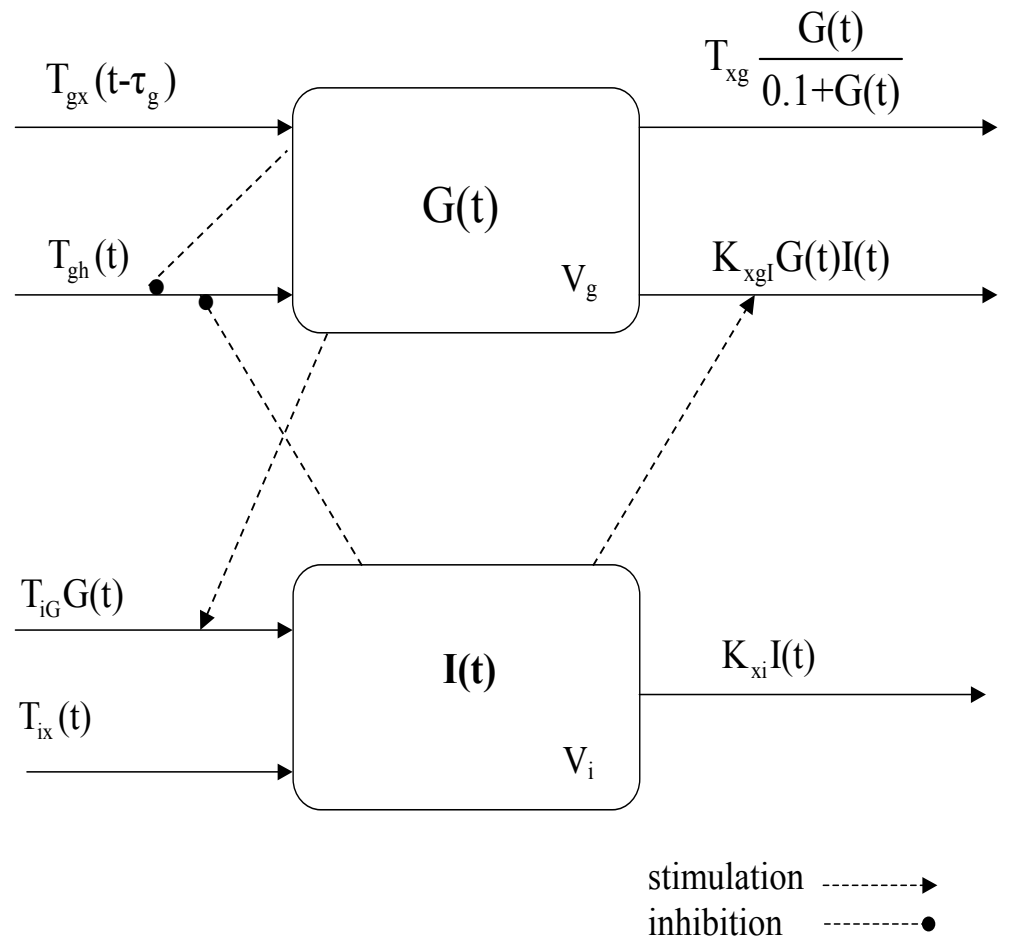

Figure 1: Schematic representation of the model (1)-(3). 


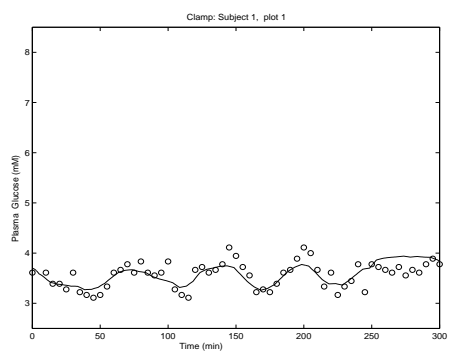

(a) Subject 1

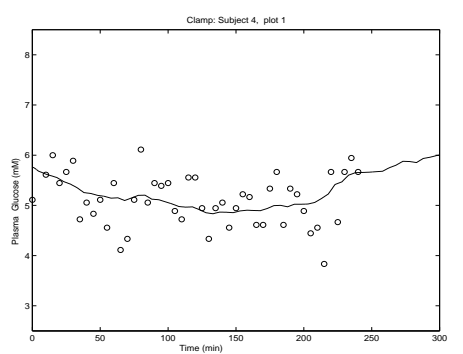

(d) Subject 4

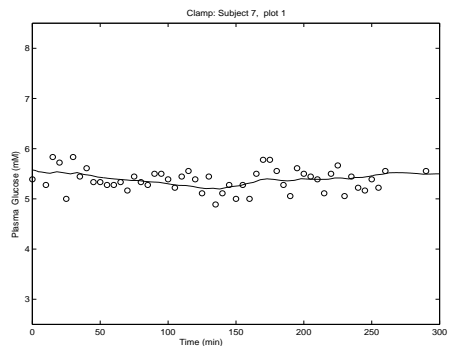

(g) Subject 7

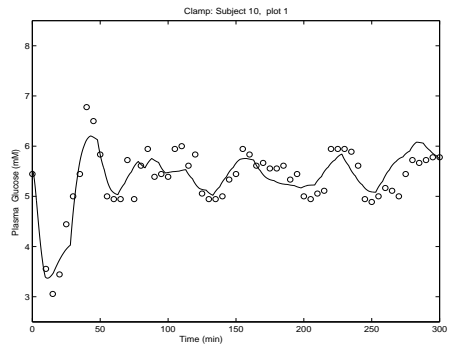

(j) Subject 10

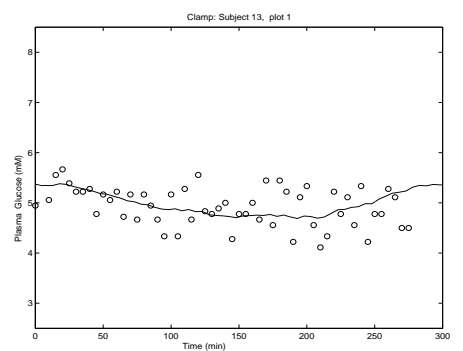

(m) Subject 13

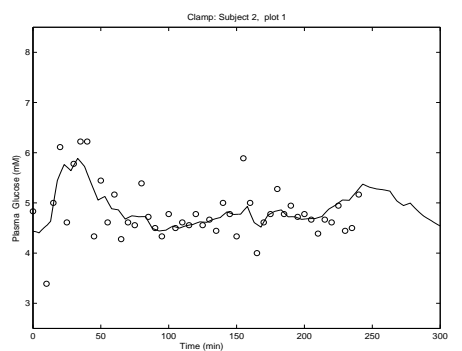

(b) Subject 2

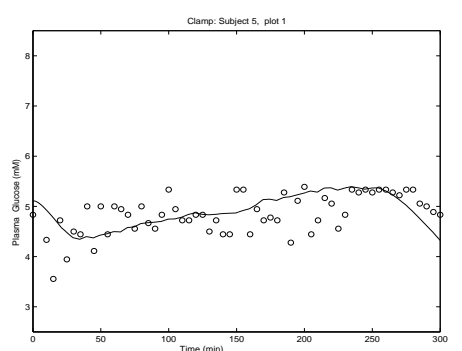

(e) Subject 5

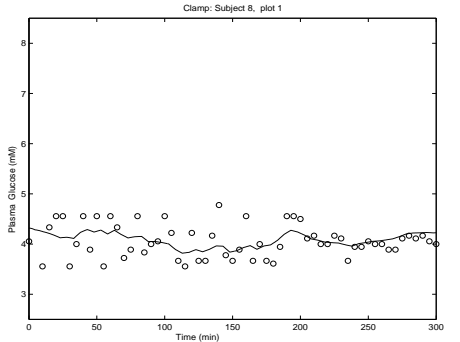

(h) Subject 8

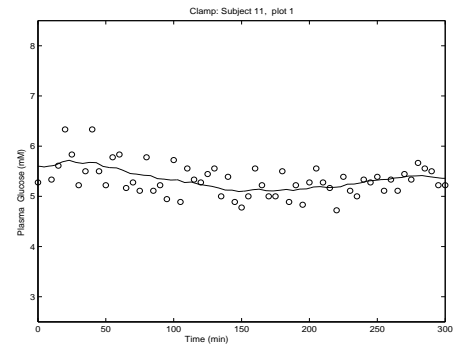

(k) Subject 11

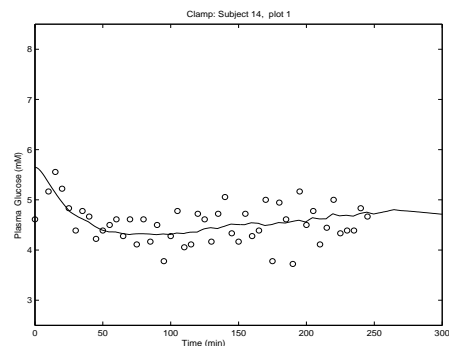

(n) Subject 14

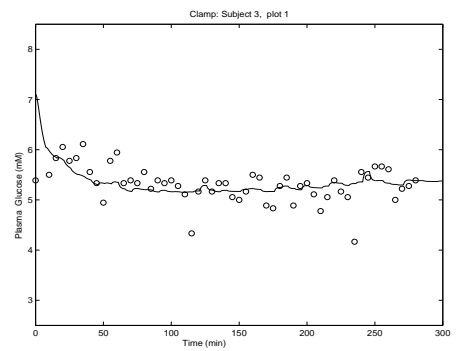

(c) Subject 3

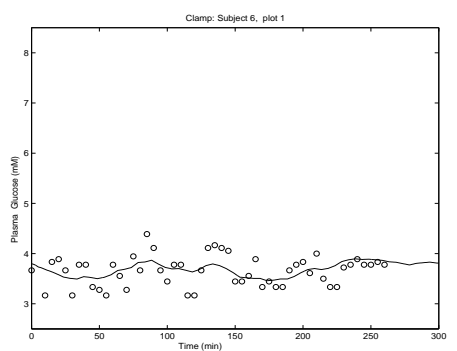

(f) Subject 6

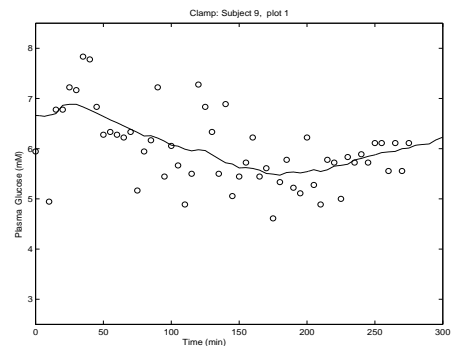

(i) Subject 9

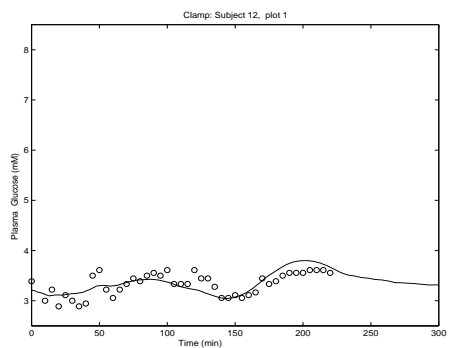

(l) Subject 12

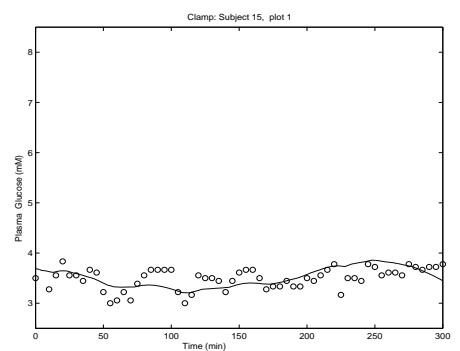

(o) Subject 15

Figure 2: ODE model: observed (o) and predicted (solid line) glycemia corresponding to the IRWLS estimates for the case $\left(C V_{G}, C V_{I}\right)=(0.05,0.15)$ (see Table 5$)$. 


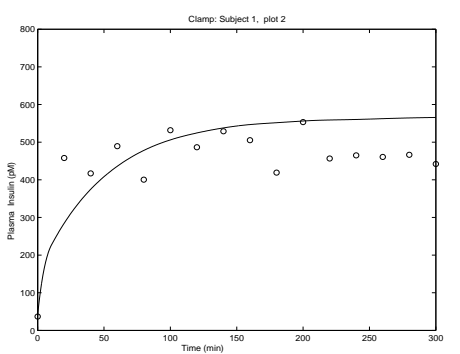

(a) Subject 1

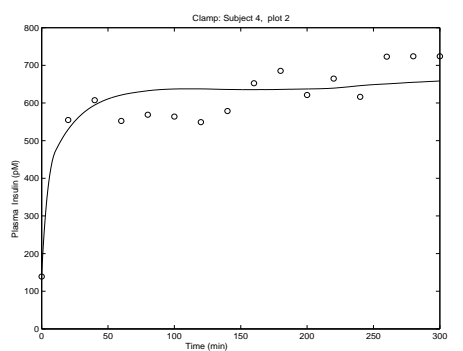

(d) Subject 4

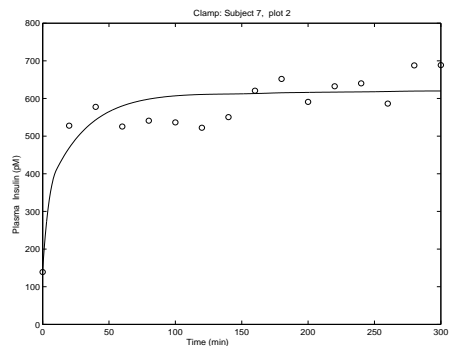

(g) Subject 7

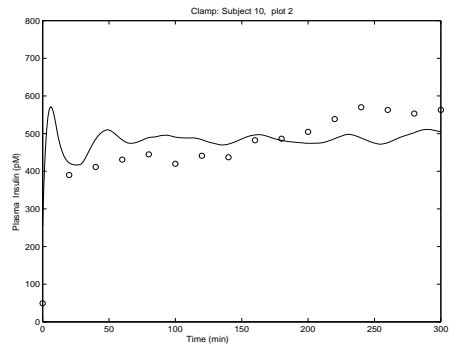

(j) Subject 10

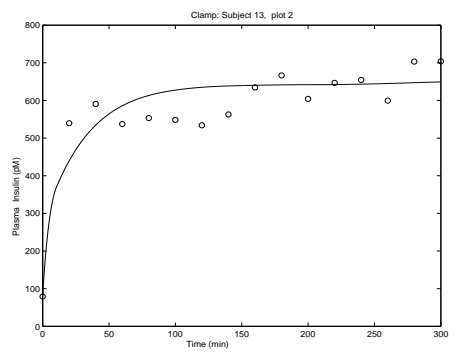

(m) Subject 13

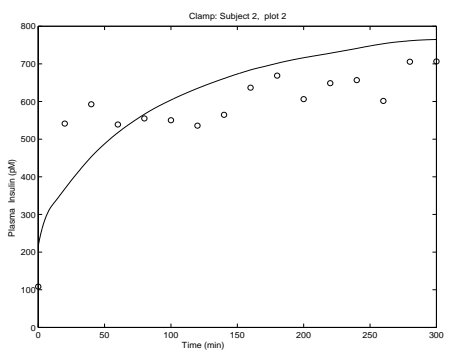

(b) Subject 2

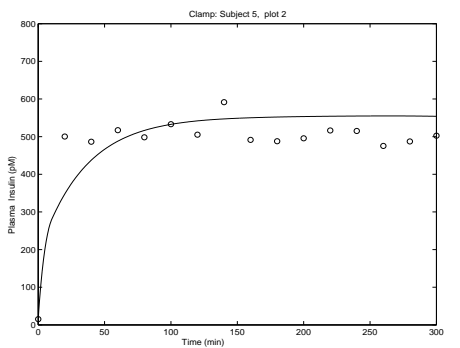

(e) Subject 5

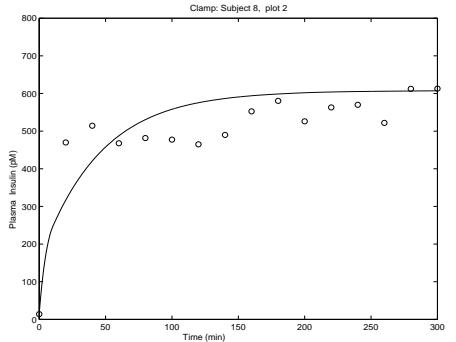

(h) Subject 8

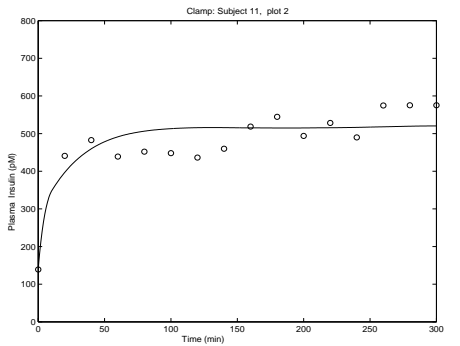

(k) Subject 11

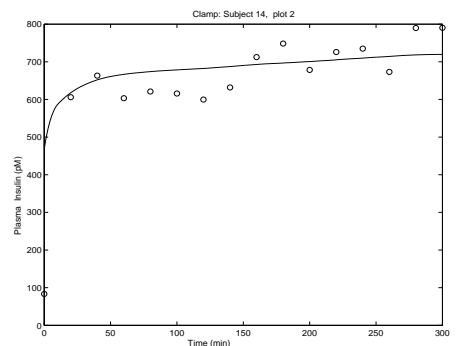

(n) Subject 14

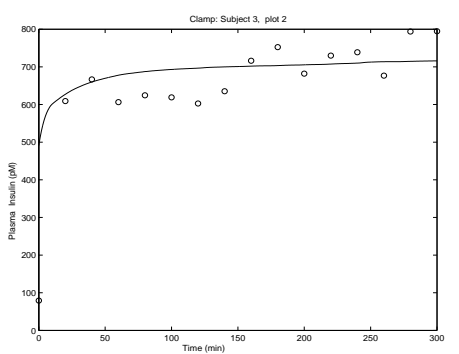

(c) Subject 3

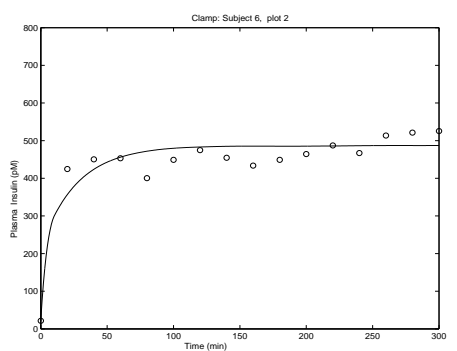

(f) Subject 6

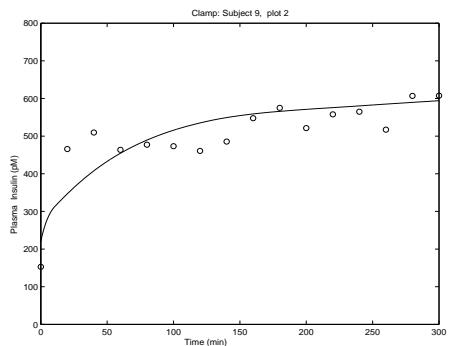

(i) Subject 9

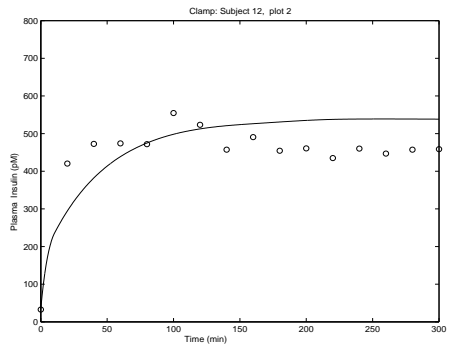

(l) Subject 12

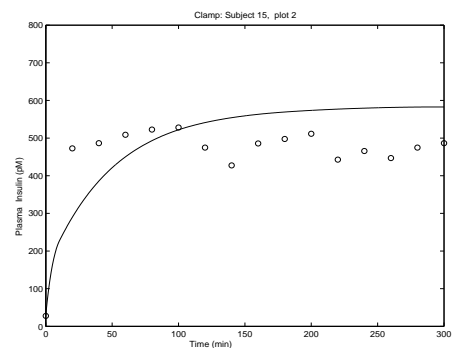

(o) Subject 15

Figure 3: ODE model: observed (o) and predicted (solid line) insulinemia corresponding to the IRWLS estimates for the case $\left(C V_{G}, C V_{I}\right)=(0.05,0.15)$ (see Table 5$)$. 


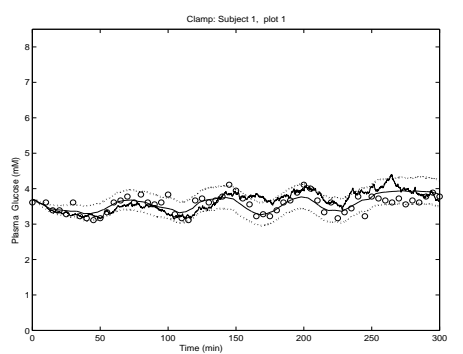

(a) Subject 1

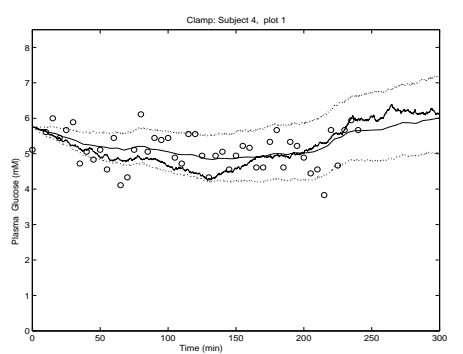

(d) Subject 4

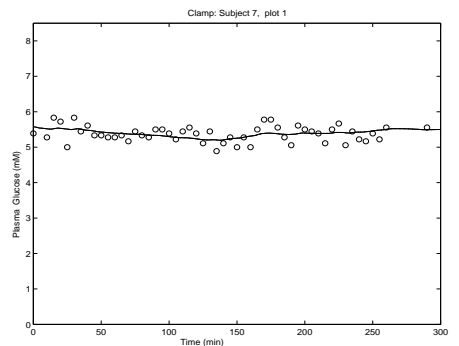

(g) Subject 7

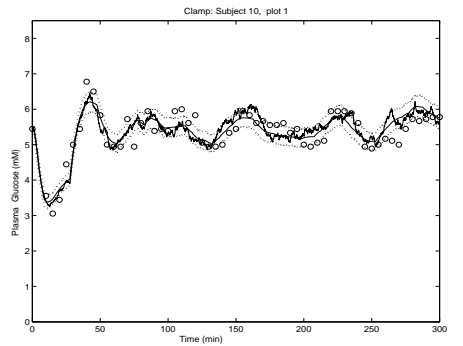

(j) Subject 10

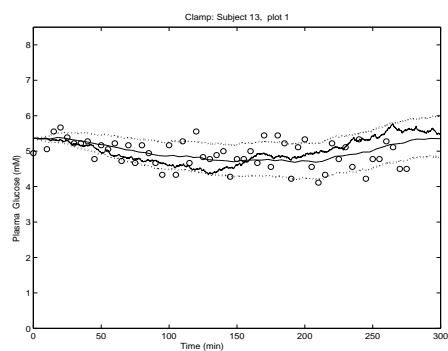

(m) Subject 13

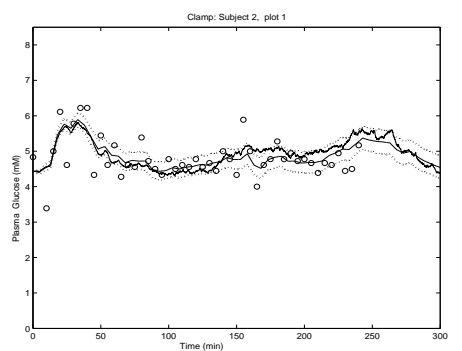

(b) Subject 2

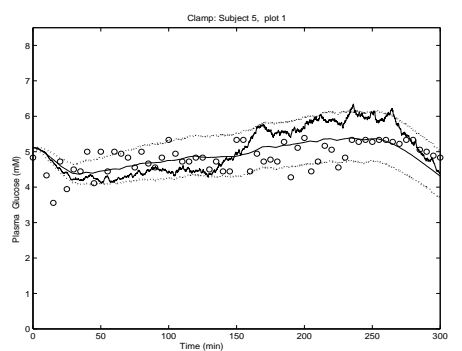

(e) Subject 5

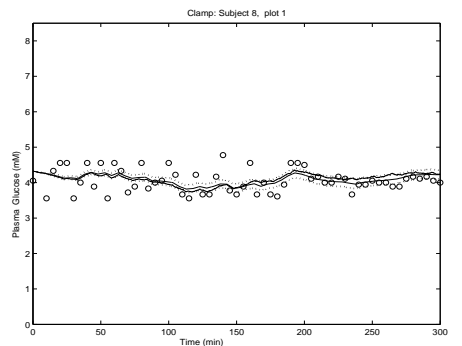

(h) Subject 8

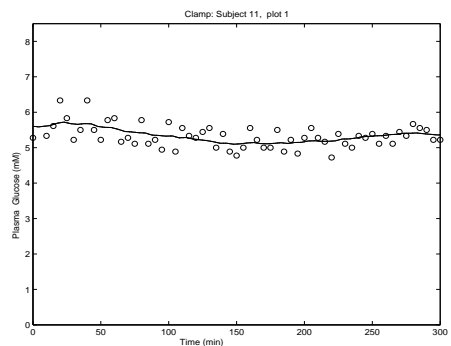

(k) Subject 11

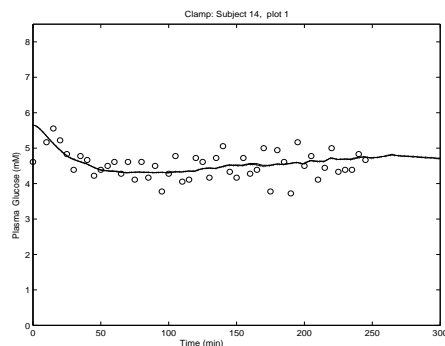

(n) Subject 14

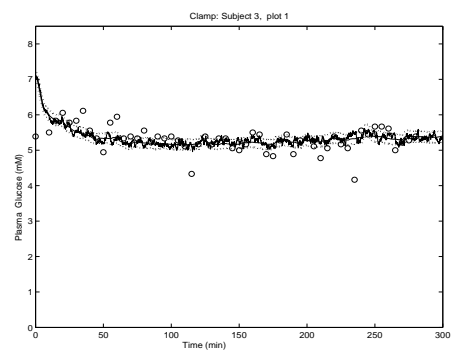

(c) Subject 3

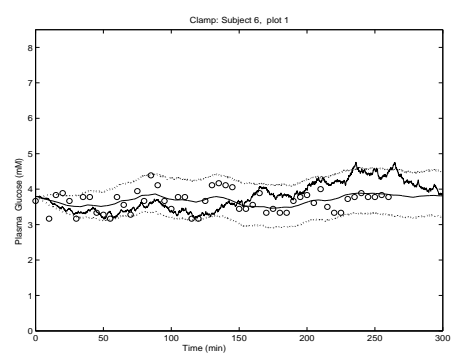

(f) Subject 6

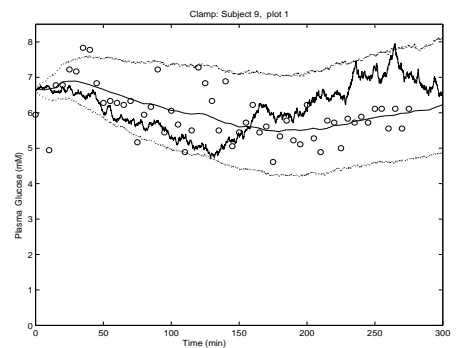

(i) Subject 9

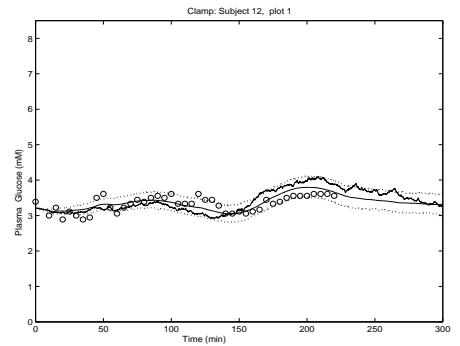

(l) Subject 12

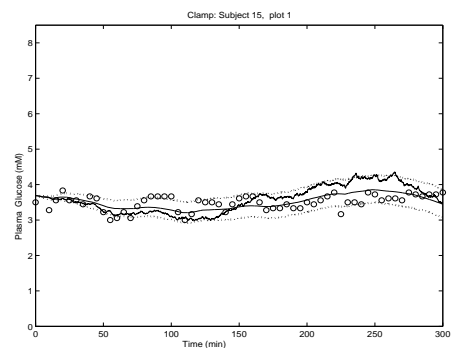

(o) Subject 15

Figure 4: SDE model: a simulated trajectory of $G(t)$, empirical mean curve of the $G(t)$ process (smooth solid lines), empirical $95 \%$ confidence limits of the mean process (dashed lines) for the case $\left(C V_{G}, C V_{I}\right)=(0.05,0.15)$ and glycemia observations. 


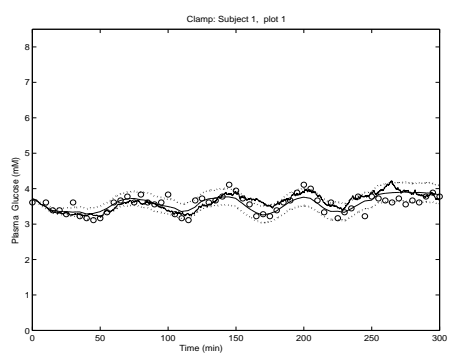

(a) Subject 1

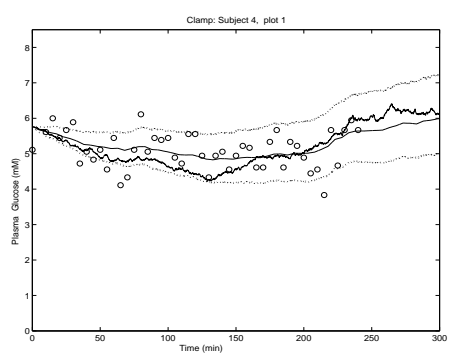

(d) Subject 4

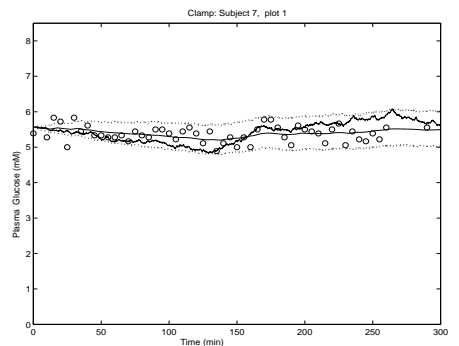

(g) Subject 7

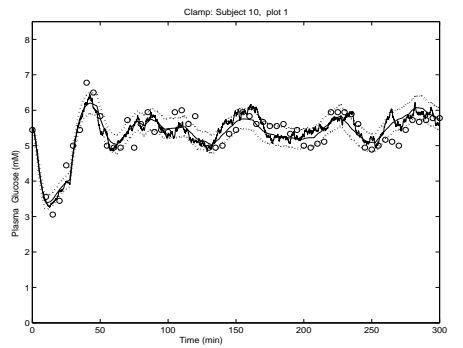

(j) Subject 10

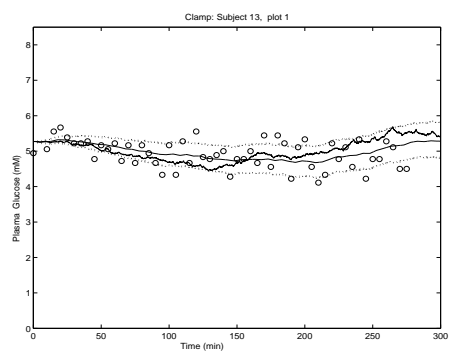

(m) Subject 13

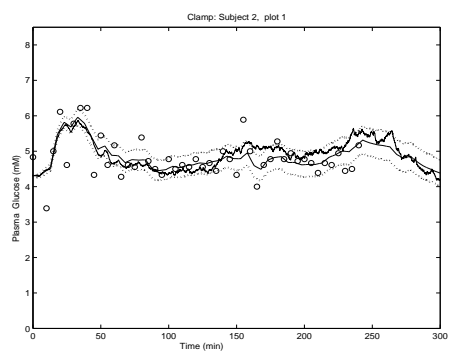

(b) Subject 2

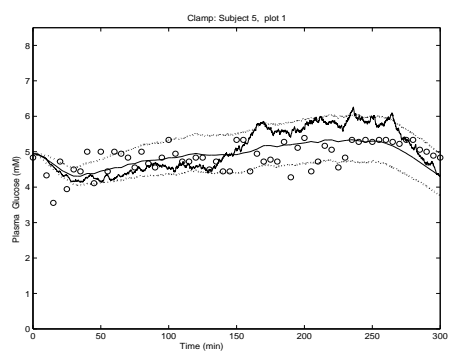

(e) Subject 5

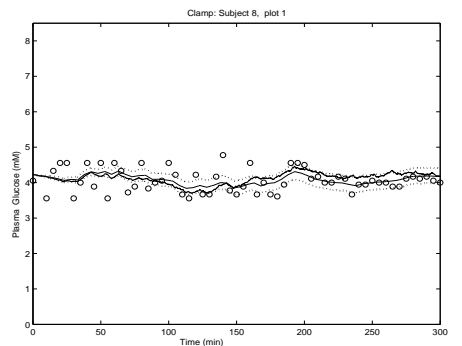

(h) Subject 8

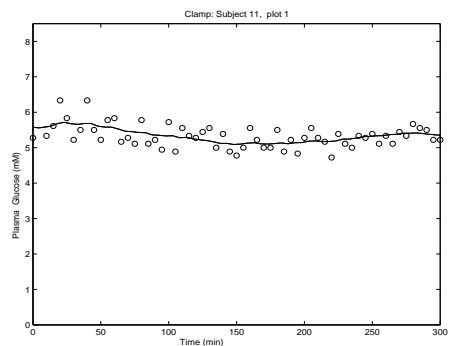

(k) Subject 11

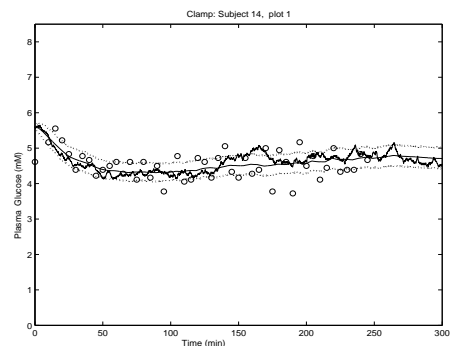

(n) Subject 14

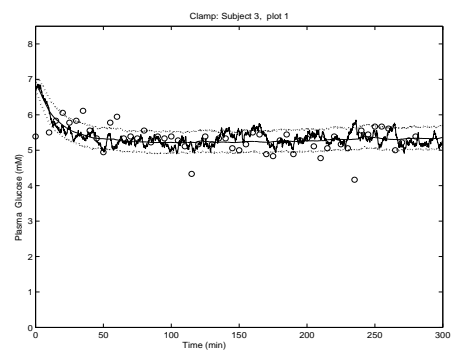

(c) Subject 3

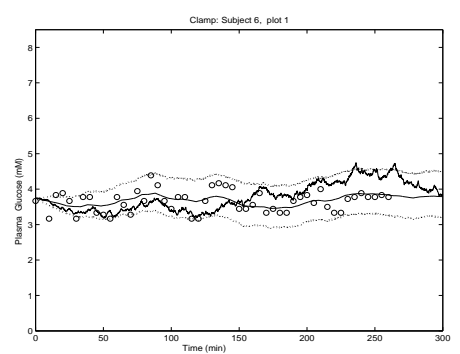

(f) Subject 6

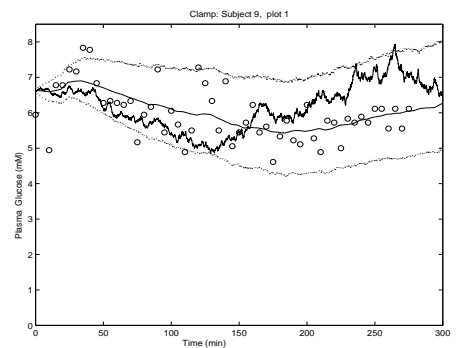

(i) Subject 9

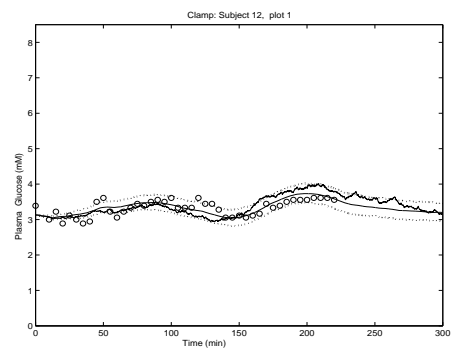

(l) Subject 12

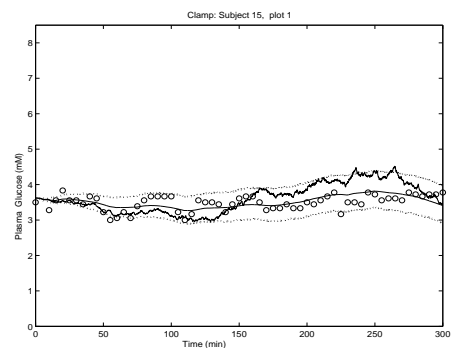

(o) Subject 15

Figure 5: SDE model: a simulated trajectory of $G(t)$, empirical mean curve of the $G(t)$ process (smooth solid lines), empirical $95 \%$ confidence limits of the mean process (dashed lines) for the case $\left(C V_{G}, C V_{I}\right)=(0.03,0.15)$ and glycemia observations. 


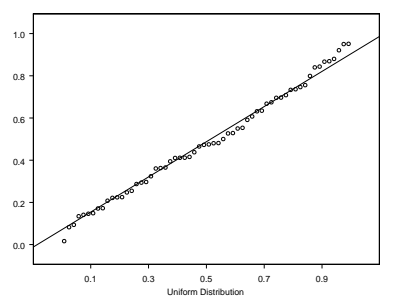

(a) Subject 1, $p=0.658$

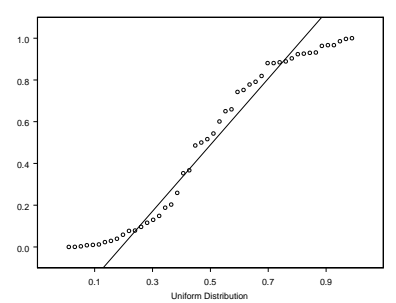

(d) Subject 4, $p=0.0485$

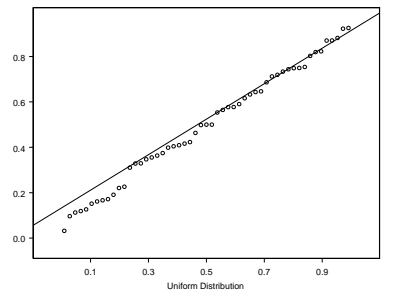

(g) Subject 7, $p=0.7241$

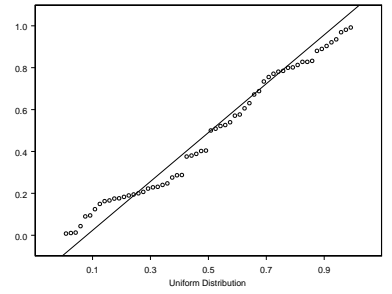

(j) Subject 10, $p=0.2676$

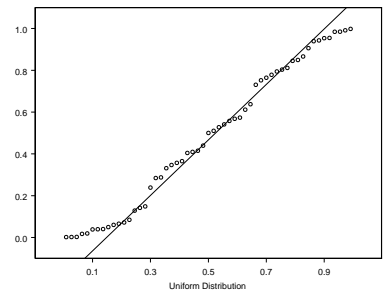

(m) Subject 13, $p=0.1585$

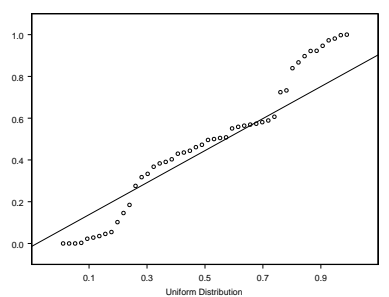

(b) Subject 2, $p=0.2545$

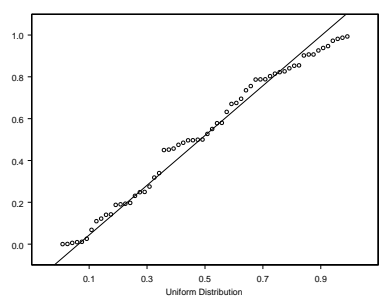

(e) Subject $5, p=0.3502$

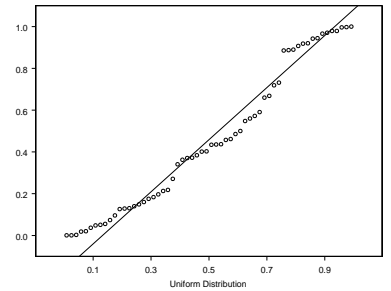

(h) Subject 8, $p=0.1418$

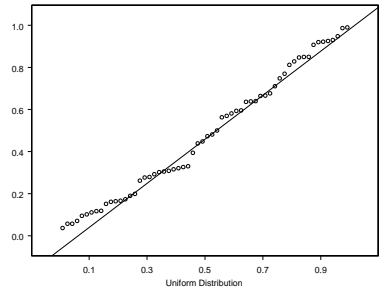

(k) Subject 11, $p=0.3553$

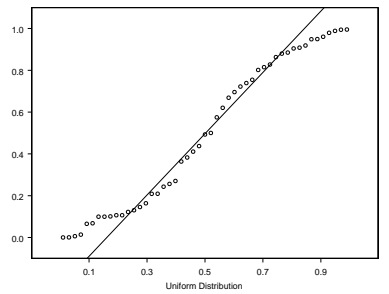

(n) Subject $14, p=0.2475$

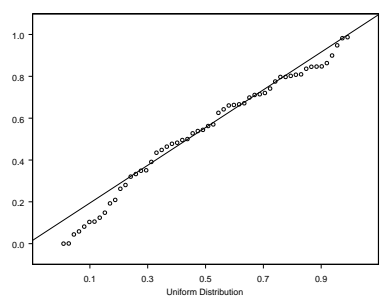

(c) Subject 3, $p=0.4677$

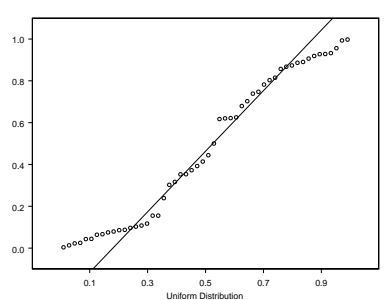

(f) Subject 6, $p=0.0452$

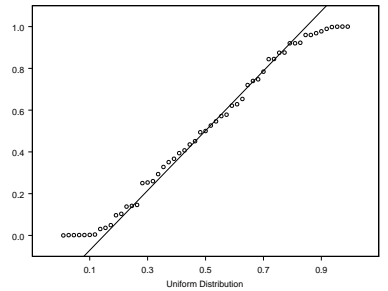

(i) Subject $9, p=0.2443$

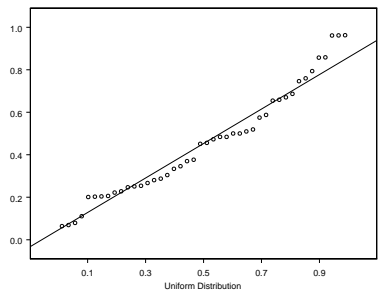

(l) Subject 12, $p=0.1732$

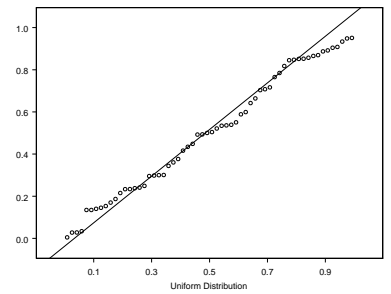

(o) Subject 15, $p=0.2545$

Figure 6: SDE model: q-q plots of the simulated glycemia residuals in the case $\left(C V_{G}, C V_{I}\right)=(0.05,0.15)$ and $p$-values from the Kolmogorov-Smirnov goodness-of-fit test. If $p<0.05$ the residuals do not conform the hypothesis of $U(0,1)$ distribution at a $5 \%$ confidence level. 


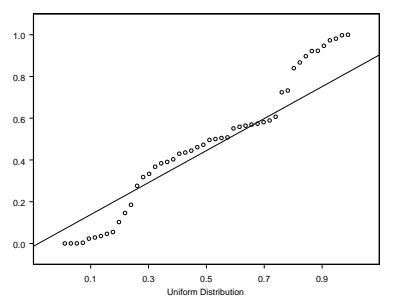

(a) Subject 1, $p=0.0922$

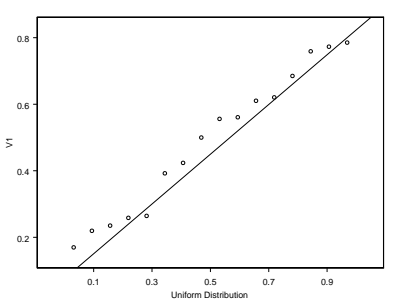

(d) Subject 4, $p=0.3954$

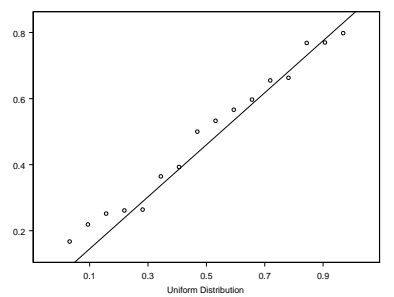

(g) Subject 7, $p=0.4722$

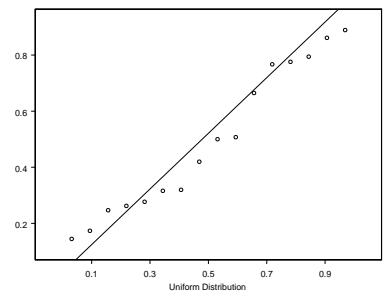

(j) Subject 10, $p=0.8462$

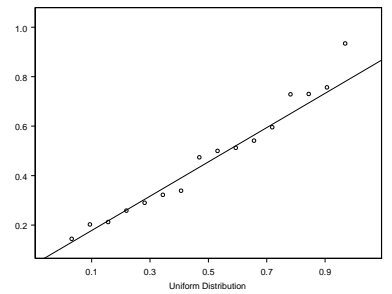

(m) Subject 13, $p=0.6089$

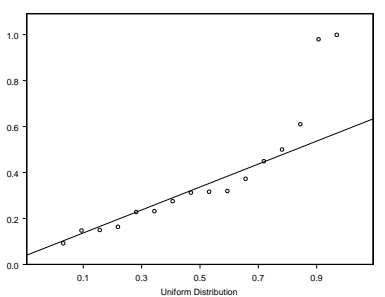

(b) Subject 2, $p=0.0657$

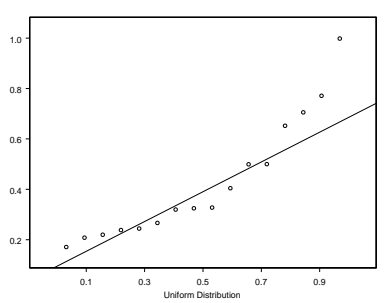

(e) Subject $5, p=0.27$

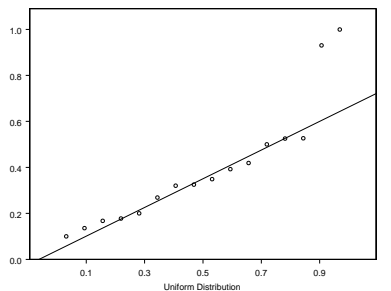

(h) Subject 8, $p=0.0305$

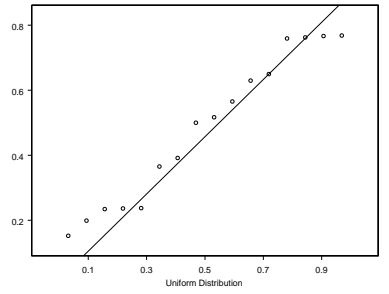

(k) Subject 11, $p=0.3077$

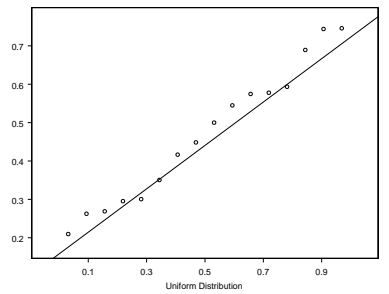

(n) Subject 14, $p=0.2132$

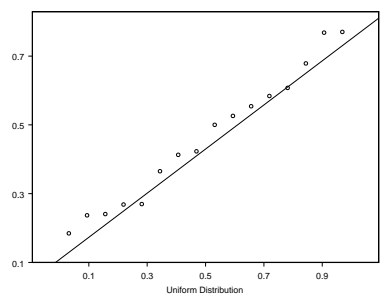

(c) Subject 3, $p=0.3172$

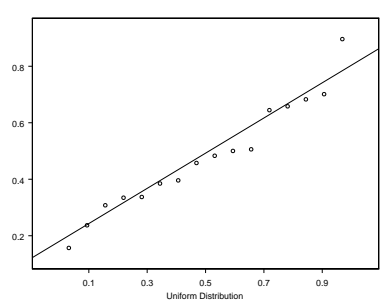

(f) Subject 6, $p=0.2847$

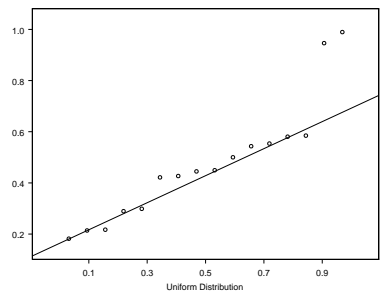

(i) Subject $9, p=0.1096$

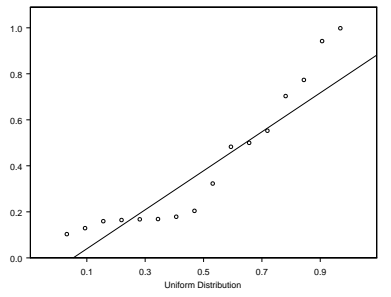

(l) Subject 12, $p=0.0981$

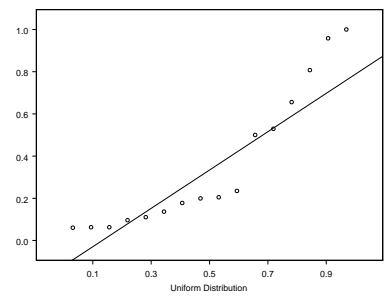

(o) Subject 15, $p=0.0105$

Figure 7: SDE model: q-q plots of the simulated insulinemia residuals in the case $\left(C V_{G}, C V_{I}\right)=(0.05,0.15)$ and $p$-values from the Kolmogorov-Smirnov goodness-of-fit test. If $p<0.05$ the residuals do not conform the hypothesis of $U(0,1)$ distribution at a $5 \%$ confidence level. 